\title{
PLCK G165.7+67.0: Analysis of a Massive Lensing Cluster in a Hubble Space Telescope Census of Submillimeter Giant Arcs Selected Using Planck/Herschel
}

\author{
Brenda L. Frye ${ }^{1}$ (D) , Massimo Pascale ${ }^{1}$, Yujing Qin ${ }^{1}$, Adi Zitrin ${ }^{2}$ (D) José Diego $^{3}$, Greg Walth ${ }^{4}$ (D) Haojing Yan ${ }^{5}$ (D), \\ Christopher J. Conselice ${ }^{6}(10)$, Mehmet Alpaslan ${ }^{7}$ (1) , Adam Bauer ${ }^{1}$, Lorenzo Busoni ${ }^{8}$, Dan Coe ${ }^{9}$, Seth H. Cohen ${ }^{10}$, Hervé Dole ${ }^{11}$, \\ Megan Donahue $^{12}$ (1), Iskren Georgiev ${ }^{13}$, Rolf A. Jansen ${ }^{10}$ (D), Marceau Limousin ${ }^{14}$, Rachael Livermore ${ }^{15}$ (1), Dara Norman ${ }^{16}$, \\ Sebastian Rabien ${ }^{17}$, and Rogier A. Windhorst ${ }^{10}$ (iD \\ ${ }^{1}$ Department of Astronomy/Steward Observatory, 933 North Cherry Avenue, University of Arizona, Tucson, AZ 85721, USA; bfrye@as.arizona.edu \\ ${ }^{2}$ Physics Department, Ben-Gurion University of the Negev, P.O. Box 653, Be'er-Sheva, 8410501, Israel \\ ${ }^{3}$ IFCA, Instituto de Fisica de Cantabria (UC-CSIC), Av. de Los Castros s/n, E-39005 Santander, Spain \\ ${ }^{4}$ Center for Astrophysics \& Space Sciences, University of California at San Diego, 9500 Gilman Drive, La Jolla, CA 92093-0424, USA \\ ${ }^{5}$ Department of Physics and Astronomy, University of Missouri, Columbia, MO 65211, USA \\ ${ }_{7}^{6}$ School of Physics and Astronomy, The University of Nottingham, University Park, Nottingham, NG7 2RD, UK \\ ${ }^{7}$ Center for Cosmology and Particle Physics, New York University, 726 Broadway, New York, NY 10003, USA \\ ${ }^{8}$ Osservatorio Astrofisico di Arcetri, Largo Enrico Fermi 5, I-50125, Florence, Italy \\ ${ }^{9}$ STScI, 3700 San Martin Drive, Baltimore, MD 21218, USA \\ ${ }^{10}$ School of Earth \& Space Exploration, Arizona State University, Tempe, AZ 85287-1404, USA \\ ${ }^{11}$ Institut d'Astrophysique Spatiale, CNRS, Univ. Paris-Sud, Université Paris-Saclay, F-91400 Orsay, France \\ ${ }^{12}$ Physics \& Astronomy Department, Michigan State University, East Lansing, MI 48824-2320, USA \\ ${ }^{13}$ Max Planck Institut für Astronomie, Königstuhl 17, D-69117, Heidelberg, Germany \\ ${ }^{14}$ Aix Marseille Université, LAM, Laboratoire d'Astrophysique de Marseille, Marseille, France \\ ${ }^{15}$ School of Physics, University of Melbourne, Melbourne, Victoria, Australia \\ ${ }^{16}$ National Optical Astronomical Observatory, 950 N. Cherry Ave., Tucson, AZ 85719, USA \\ ${ }_{17}$ Max Planck Institut für Extraterrestrische Physik, Garching, Germany \\ Received 2018 May 10; revised 2018 October 18; accepted 2018 November 7; published 2019 January 21
}

\begin{abstract}
We present Hubble Space Telescope WFC3-IR imaging in the fields of six apparently bright dusty star-forming galaxies (DSFGs) at $z=2-4$ identified by their rest-frame far-infrared colors using the Planck and Herschel space facilities. We detect near-infrared counterparts for all six submillimeter sources, allowing us to undertake stronglensing analyses. One field in particular stands out for its prominent giant arcs, PLCK G165.7+67.0 (G165). After combining the color and morphological information, we identify 11 sets of image multiplicities in this one field. We construct a strong-lensing model constrained by this lensing evidence, which uncovers a bimodal spatial mass distribution, and from which we measure a mass of $(2.6 \pm 0.11) \times 10^{14} M_{\odot}$ within $\sim 250 \mathrm{kpc}$. The bright $\left(S_{350} \approx 750 \mathrm{mJy}\right)$ DSFG appears as two images: a giant arc with a spatial extent of 4 .' 5 that is merging with the critical curve, and a lower-magnification counterimage that is detected in our new longer-wavelength ground-and space-based imaging data. Using our ground-based spectroscopy, we calculate a dynamical mass of $1.3_{-0.70}^{+0.04} \times 10^{15} M_{\odot}$ to the same fixed radius, although this value may be inflated relative to the true value if the velocity distribution is enhanced in the line-of-sight direction. We suggest that the bimodal mass taken in combination with the weak X-ray flux and low SZ decrement may be explained as a pre-merger for which the intracluster gas is diluted along the line of sight, while the integrated surface mass density is supercritical to strong-lensing effects.
\end{abstract}

Key words: galaxies: clusters: general - galaxies: fundamental parameters - galaxies: high-redshift - gravitational lensing: strong - submillimeter: galaxies

\section{Introduction}

Clusters of galaxies with masses $\sim 10^{15} M_{\odot}$ are extremely useful but rare tracers of the distribution of mass in the universe (Bahcall 1977; Mo \& White 1996). Finding the galaxy clusters and then establishing their cluster properties and cluster scaling relations are fundamental to cosmology studies (Vikhlinin et al. 2009; Mantz et al. 2010; Rozo et al. 2010; Allen et al. 2011; Benson et al. 2013; Hasselfield et al. 2013; Planck Collaboration et al. 2014). As ensembles of discrete galaxies, clusters can be discovered in optical and near-infrared (NIR) wide-area surveys, such as the Sloan Digital Sky Survey (SDSS; i.e., Koester et al. 2007a, 2007b; Rykoff et al. 2014, 2016).

Although originally discovered at optical wavelengths, galaxy clusters with masses of $(1-10) \times 10^{15} M_{\odot}$ will almost always contain a massive component of hot intracluster gas, which makes them distinct X-ray sources. This reservoir of hot baryons is a salient feature of massive clusters, as there is no physical mechanism to dissipate it. To search for this requisite feature, the ROSAT archives offer the all-sky advantage to efficiently detect the most extreme sources of X-ray emission produced by thermal bremsstrahlung and line emission in the intracluster gas (Rosati et al. 1998; Ebeling et al. 2007, 2010).

A galaxy cluster bound by gravity also has a distinct signature at radio wavelengths. This is because the same large reservoirs of intracluster gas that give rise to the X-ray flux also distort the cosmic microwave background (CMB) radiation by inverse Compton scattering. From the ground, searches for galaxy clusters by the detection of this Sunyaev-Zel'dovich (SZ) effect using the South Pole Telescope (SPT; Carlstrom et al. 2011) yield hundreds of candidates (Bleem et al. 2015). Targeted searches using the Atacama Cosmology Telescope (ACT; Fowler et al. 2007) that exercise a similar approach are also successful (Sehgal et al. 2011, 2013). From space, Planck High Frequency Imager (HFI) data are used to extend the 
search for the SZ decrement to all available extragalactic sky (Lamarre et al. 2003; Planck Collaboration et al. 2016).

To complement the cosmological SZ approach of searching for clusters, the detection of apparently bright galaxies by the astronomical technique of color selection has recently been explored. For example, the infrared-bright dusty star-forming galaxies (DSFGs) produce stars at rates of up to $\sim 1000$ $M_{\odot} \mathrm{yr}^{-1}$ and yield prodigious amounts of dust. This warm dust radiates as a modified blackbody spectrum with a prominent peak in the rest-frame far-infrared. Submillimeter data are well suited to conduct the color search for the DSFGs, because this wavelength range corresponds to the observed-frame thermal dust peak at redshifts typical of DSFGs of $z \approx 2-4$ (Casey et al. 2014; Planck Collaboration 2015, and references therein). In this regime, there is the unusual advantage that one records the flux density of the DSFGs closer to the peak of their rest-frame spectral energy distributions (SEDs) as their redshift increases. As a result, the benefit of the high flux density of DSFGs largely compensates for the cosmological dimming (Blain 1999; Planck Collaboration 2015), thereby gaining leverage for the detection of high-redshift objects.

A Planck/HFI (Planck Collaboration et al. 2016) census was undertaken to find DSFGs by color covering the portion of the sky with minimal cirrus contamination, which we take to mean that the column density of hydrogen in the $857 \mathrm{GHz}$ map is less than the minimum value of $N_{\mathrm{H} \mathrm{I}}=3 \times 10^{20} \mathrm{~cm}^{-1}$, amounting to $26 \%$ of the sky. To be selected by Planck/HFI, the DSFGs had to be separately detected in each of the cleaned 857, 545 and $353 \mathrm{GHz}$ maps, be compact at Planck resolution $\left(\sim 4.5^{\prime}\right)$, and have flux density ratios in the 353,545 , and $857 \mathrm{GHz}$ maps consistent with being red and dusty sources (Planck Collaboration 2015). These so-called "cold" sources of the cosmic infrared background (CIB; Puget et al. 1996; Dole et al. 2006) are extremely rare at the Planck point-source sensitivity of about $\sim 600 \mathrm{mJy}$ at $\sim 545 \mathrm{GHz}$, with number densities of $\sim 1$ per few square degrees, requiring the wide-field survey area of Planck/HFI. Planck Collaboration (2016) selected 2000 DSFG candidates this way.

To classify the sources, Planck Collaboration (2015) performed follow-up observations at higher angular resolution using the Herschel/SPIRE (Pilbratt et al. 2010) on a subset of the Planck candidate DSFGs, consisting of the 228 brightest Planck sources. Details and initial results are discussed elsewhere (Planck Collaboration 2015, 2016). Relevantly, 15 of the 228 sources are discovered to be individual DSFGs boosted in brightness as a result of gravitational lensing (Cañameras et al. 2015; Planck Collaboration 2015).

Herein we present new Hubble Space Telescope (HST) imaging and lensing analysis for 6 of the 15 strongly lensed Planck/Herschel-selected sources. We expand our study about one particular field in our sample, namely, PLCK G165.7+67.0 (hereafter G165), which shows strong-lensing constraints in the form of giant arcs and image multiplicities. To better understand the properties of this one field, we acquire multiwavelength imaging and spectroscopic follow-up observations, which will be discussed in detail.

This paper is organized as follows. In Section 2 we compare our sample of strongly lensed DSFGs with others in the literature. In Section 3, we present new HST imaging data for our sample of six Planck/Herschel-selected strongly lensed DSFGs. We also present new ground- and space-based observations of G165. In Section 4, we describe the data reduction and analysis

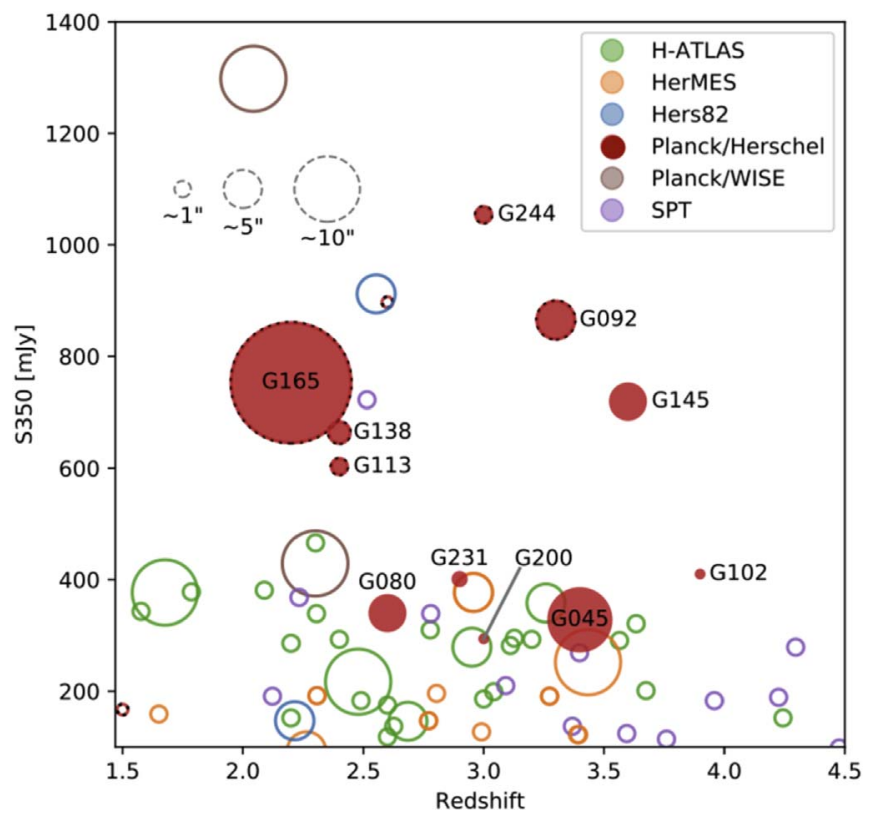

Figure 1. $S_{350}$ flux density of lensed DSFGs for the Planck/Herschel-selected sample (filled red disks; Cañameras et al. 2015), complemented by others from the literature (open circles). Our sample partially intersects with another survey of Planck/Herschel-selected sources (black-and-red dashed circles; Harrington et al. 2016). The other lensed DSFGs indicated are sourced from the literature. These are H-ATLAS (green; Harris et al. 2012; Bussmann et al. 2013; Calanog et al. 2014; Negrello et al. 2017), HerMES (orange; Bussmann et al. 2013; Wardlow et al. 2013; Calanog et al. 2014; Nayyeri et al. 2016), Hers82 (blue; Nayyeri et al. 2016), Planck/WISE (brown; Díaz-Sánchez et al. 2017), and SPT (purple; Vieira et al. 2013; Weiß et al. 2013; Spilker et al. 2016). In all cases, the symbol size represents the Einstein radius as estimated from the resolved image of the lensed source. With some exceptions, the Planck/ Herschel selection tends to select lensed DSFGs with higher flux densities and larger Einstein radii.

of the follow-up data obtained for G165. In Section 5, we construct the strong-lensing model for G165. This analysis is followed by a discussion of G165 in Section 6, in which we make independent determinations of the mass, the lensing strength, and the properties of the low inferred cluster gas pressure. In Section 7, we summarize our results. Appendices are provided to describe the imaging and lensing analysis of all six fields in our $H S T$ sample. We assume throughout a $\Lambda \mathrm{CDM}$ cosmology with $H_{0}=67 \mathrm{~km} \mathrm{~s}^{-1} \mathrm{Mpc}^{-1}, \Omega_{m, 0}=0.32$, and $\Omega_{\Lambda, 0}=0.68$ (Planck Collaboration et al. 2018).

\section{Strongly Lensed DSFGs}

Although the details of the search strategies for strongly lensed DSFGs differ, most algorithms set a high $350 \mu \mathrm{m}$ flux density $\left(S_{350}\right)$, or a high $500 \mu \mathrm{m}$ flux density $\left(S_{500}\right)$, cut of $100 \mathrm{mJy}$. To date, dozens of strongly lensed DSFGs in the redshift range $2<z<4$ satisfy these criteria. In Figure 1, we assemble the set of lensed DSFGs for the surveys, or subsets thereof, for which there are Planck $S_{350}$ flux densities, spectroscopic redshifts for the lens and the lensed sources, reported Einstein radii, and images of the lensed sources. For each DSFG in Figure 1, the symbol size is proportional to the size of the Einstein radius. For our sample, we measured the Einstein radius at the source redshift using our light-tracesmass model (Zitrin et al. 2009, 2015). For the objects in other samples, we estimated the Einstein radius by eye from the resolved image of the lensed source, or by a table made available to us for the case of the SPT sources (Spilker, private 
communication). If a resolved image was not supplied for a lensed source, then it is not included in Figure 1. For reference, the Einstein radii are assigned to either a $1^{\prime \prime}, 5^{\prime \prime}$, or $10^{\prime \prime}$ bin. These bins are used to distinguish the scale of the lens type approximately as a massive galaxy lens $\left(M \sim 10^{11} M_{\odot}\right)$, a galaxy group lens $\left(M \sim 10^{13} M_{\odot}\right)$, or galaxy cluster lens $\left(M \sim 10^{15} M_{\odot}\right)$, respectively. The legend gives the color-coded references and the bin sizes. The brightest lensed DSFG, the "Cosmic Eyebrow" $(z=2.0439)$, stands out for its high submillimeter flux density $\left(S_{350}=1298 \pm 200 \mathrm{mJy}\right)$. It was found by cross-correlating the sources in the WISE all-sky source catalog "AllWISE," with infrared-bright galaxies in the Planck compact source catalog (single brown circle in Figure 1; Díaz-Sánchez et al. 2017). Note that the submillimeter flux density of the Cosmic Eyebrow is measured from Planck/HFI data, which have a higher uncertainty than the Herschel/SPIRE photometry used for the other comparison samples in Figure 1. Even if the true value is closer to its lower limit, it would still be the brightest known DSFG at $350 \mu \mathrm{m}$.

In the redshift range $2<z<4$, a search for lensed DSFGs within the Herschel Astrophysical Terahertz Large Area Survey (H-ATLAS) using $S_{500}$ as a discriminator yields 22 lensed DSFGs covering a search area of $14.4 \mathrm{deg}^{2}$ (Harris et al. 2012; Bussmann et al. 2013; Calanog et al. 2014; Negrello et al. 2017). This same approach applied to the Herschel Multitiered Extragalactic Survey (HerMES) field extends the areal coverage by a factor of $\sim 7$, resulting in 13 new lensed sources (Bussmann et al. 2013; Wardlow et al. 2013; Calanog et al. 2014; Nayyeri et al. 2016). By applying similar flux density cuts to the Herschel Stripe 82 Survey (Hers82), an additional three lensed DSFGs are found (Nayyeri et al. 2016).

From the ground, South Pole Telescope (SPT) data enable the selection of strongly lensed DSFGs based on the ratio of flux densities at 1.4 and $2.0 \mathrm{~mm}, S_{1.4 \mathrm{~mm}} / S_{2 \mathrm{~mm}}$, which are consistent with thermal emission by dusty galaxies (Vieira et al. 2010; Carlstrom et al. 2011). The brightest sources in the sample are then followed up at higher resolution using most notably the Submillimeter Array (SMA) and the Atacama Large Millimeter Array (ALMA). A total of 26 strongly lensed DSFGs are identified, which tend to be at higher redshifts owing to their selection at longer wavelengths, and whose identifications are typically explained as galaxy-galaxy lensing events (Weiß et al. 2013; Vieira et al. 2013).

From space, Planck and Herschel data are used to extend the search for lensed DSFGs to all available sky. The search technique relies on the detection of the rest-frame far-infrared thermal dust peak, which is a salient feature of DSFGs. A strict lower limit on the flux density is imposed, amounting to $600 \mathrm{mJy}$ at $545 \mathrm{GHz}$, to select only the most extreme sources. The expectation is that the brightest sources that are also compact at Planck resolution and that remain compact in the Herschel follow-up observations are too faint to be explained by a single-field DSFG. These sources are most likely (1) multiple DSFGs or (2) a single strongly lensed DSFG. At the higher resolution using Herschel/SPIRE, the vast majority of sources resolve out into clumps of several submillimeter-bright objects in close projected proximity (Planck Collaboration 2015, 2016). These are the candidate galaxy overdense regions, which are potentially the highredshift predecessors of massive lensing clusters at lower redshifts (Planck Collaboration 2015; Flores-Cacho et al. 2016; Martinache et al. 2018; Kneissl et al. 2018).
At the same time, a small minority of 15 of 228 sources remained isolated, while also meeting additional flux density thresholds of $S_{350}>300 \mathrm{mJy}$ and/or $S_{500}>300 \mathrm{mJy}$. These sources show signatures of individual DSFGs that are boosted in brightness as a result of strong lensing. Of these, 11 sources could be followed up at higher resolution using observing facilities from the Northern Hemisphere. Spectroscopic measurements of the lens and source redshifts and identification of giant arc structures strengthen their lensing interpretation (filled red circles in Figure 1; Cañameras et al. 2015, 2017a, 2017b; Nesvadba et al. 2016).

The Harrington et al. (2016) sample (black-and-red dashed circles) is closely related to the Cañameras et al. (2015) sample. Their selection also relies on color using a combination of Planck and Herschel, yet the intersection is incomplete owing to the use of different Planck catalogs. Harrington et al. (2016) select sources by cross-correlating Herschel/SPIRE with Planck PCCS (six candidates), Planck HerMes (one candidate), and Planck Hers82 (one candidate). The selection of the Planck/Herschel sample (Cañameras et al. 2015) was made by applying color criteria to Planck PCCS and Herschel/SPIRE (six candidates) and to Planck OT2 and Herschel/SPIRE (five candidates). In all, three of eight of the Harrington et al. (2016) lensed DSFGs are new.

In conclusion, there is a tendency for Planck/Herschelselected sources to have higher flux densities and larger Einstein radii than those drawn from the literature. The cluster scale of the lens may partially explain this difference, in that a larger magnification factor $(\mu)$ can be achieved, especially in the case of an Einstein ring such that $\mu \propto \sqrt{M_{\text {lens }}}$, where $M_{\text {lens }}$ is the mass of the lens. The wider areal coverage of a factor of $\sim 10$ relative to the SPT and a factor of $\sim 100$ or more relative to H-ATLAS, HerMES, and Hers 82 also helps by allowing to set higher flux density thresholds, resulting in the identification of larger lenses in some cases.

\section{Observations and Reduction}

We present new observations using HST/WFC3-IR for the six fields in our sample. $H S T /$ WFC3-IR provides a high spatial resolution 0 "! 16 FWHM at $1.6 \mu \mathrm{m}$ and a high sensitivity with a reported $5 \sigma$ point-source limiting magnitude in the F160W band of 27.0 AB mag (Windhorst et al. 2011). We expand our study in the field of G165, selected because it produces significantly more lensing evidence, which leads to a more robust lens model. To better characterize the additional lensing constraints in this one rich field, we acquired also new observations using LBT/LUCI + ARGOS, Spitzer/IRAC, Gemini/GMOS, and MMT/Hectospec.

\subsection{HST Observations}

We obtained imaging of six Planck/Herschel-selected fields between 2015 December and 2016 July with the HST Wide Field Camera 3 IR detector (WFC3-IR) in Cycle 23 (GO14223; PI: Frye). The fields are PLCK G145.2+50.9 (G145), PLCK G244.8+54.9 (G244), PLCK G165.7+67.0 (G165), PLCK G045.1+61.1 (G045), PLCK G080.2+49.8 (G080), and PLCK G092.5+42.9 (G092). The imaging is composed of one orbit each in the F110W and F160W filters. Table 1 gives the observing details.

The WFC3-IR images are redrizzled using the software package DrizzlePac (Fruchter et al. 2010). We adopt values for 
Table 1

The HST Sample: Observing Details

\begin{tabular}{lcc}
\hline \hline \multirow{2}{*}{ Lensing Field } & \multicolumn{2}{c}{ Exp. (s) } \\
\cline { 2 - 3 } & F110W & F160W \\
\hline PLCK G145.2+50.9 (G145) & 2808 & 2736 \\
PLCK G244.8+54.9 (G244) & 2592 & 2484 \\
PLCK G165.7+67.0 (G165) & 2664 & 2556 \\
PLCK G045.1+61.1 (G045) & 2556 & 2556 \\
PLCK G080.2+49.8 (G080) & 2664 & 2664 \\
PLCK G092.5+42.9 (G092) & 2808 & 2736 \\
\hline
\end{tabular}

the photon-sensitive effective size of a pixel to its real size (final_pixfrac) and a final pixel scale (final_scale) of 0.85 and $0 . " 06$, respectively. We checked the redrizzled images by computing the FWHM of a few stars in each field. In some cases, such as the G165 field, there were only two isolated, unsaturated stars within the field of view, so we substituted a compact and isolated elliptical galaxy as a third source. Redrizzling of the data in each case resulted in a $3 \%-10 \%$ improvement in image quality (FWHM) over the pipeline products. The final reduced images reach comparable depths to the CLASH clusters. For one representative case, G165, we compute $10 \sigma$ limiting magnitudes of $\mathrm{F} 110 \mathrm{~W}_{\mathrm{AB}}=26.9 \mathrm{mag}$ and $\mathrm{F} 160 \mathrm{~W}_{\mathrm{AB}}=26.2 \mathrm{mag}$ for point sources inside 0." 4 apertures. We find that the image depth and filters are sufficient to make NIR detections of the strongly lensed DSFG in each of our sample fields. We also identify other examples of giant arcs and/or image multiplicities in some cases. In Figure 2, we present the HST color images of the central regions for each of the six fields. We refer the reader to Appendix A for further details regarding the search for the NIR counterparts.

The WFC3 F160W images are used as detection images for the matched aperture photometry. We custom-built our code to cope with the unusual morphologies peculiar to arcs in the central regions of massive lensing clusters. We detect sources by applying a $\sigma$-clipping algorithm with respect to the local background rms values. The local background, in turn, is estimated following a similar approach to that in SExtractor (Bertin \& Arnouts 1996). Briefly, the image is divided into patches of $100 \times 100$ pixels, with the background in each patch represented by the $\sigma$-clipped median. The local background is then estimated by a smooth $3 \times 3$ spline interpolation over these patches. To ensure robust detection of objects, we smooth the image with a Gaussian filter of FWHM $\sim 0$ ". 2 . Objects are deblended using the watershed PYTHON algorithm in astropy . photutils (The Astropy Collaboration et al. 2018). Artifacts such as diffraction spikes are visually identified and removed.

We assign apertures to each galaxy image by measuring the semimajor/minor axis sizes at 3 times the FWHM lengths of the detected objects, typically amounting to 0.6 . Elliptical annuli are used to get the best estimate of the background, with an inner radius equal to the photometric aperture and an outer radius equal to 1.2 times the inner radius. We compute the aperture flux and then subtract the area-scaled local background level within the annuli. The flux uncertainty is computed as the quadratic sum of the local smooth background rms value only. We note that aperture corrections are minimal owing to our large extraction aperture of $>0$ ". 4 . In three fields, G145, G045, and G080, the multiple images of the single DSFG are typically too faint and/or blended with the halos of bright cluster members near in projection to measure a flux. In these cases, a $1 \sigma$ upper limit on their fluxes is reported. In Table 2 we present the photometric catalog of the lensed DSFGs for our sample. The magnification factor, $\mu$, of the lensed DSFG was measured using our light-traces-mass lens model. The effective Einstein radius at the redshift of the lensed source, $\theta_{E}=\sqrt{A / \pi}$, was measured from our lens model.

\subsection{LBT Observations}

We acquired imaging of G165 in $K$ band using the LBT Advanced Rayleigh Ground layer adaptive Optics System (ARGOS; Rabien et al. 2018) during instrument commissioning time in 2016 December (2016B; PI: Frye). ARGOS corrects ground-layer distortions in the imaging of the two 8.4 $\mathrm{m}$ apertures using two three-beam constellation lasers as guide stars that are fixed to each aperture. The ARGOS instrumentation operates through the LUCI imager and multislit spectrometer. High-quality corrections of up to FWHM $\approx 0$ " 25 at $K$ are achievable across a large field of view $\left(4^{\prime} \times 4^{\prime}\right)$ at a native pixel scale of 0 ". 12 pixel $^{-1}$. We acquired LBT/LUCI + ARGOS data in monocular mode on two separate nights: 46 minutes of observation using LUCI2 on 9 December and 42 minutes using LUCI1 on 2016 December 15 . We note that the LUCI1 set of observations have a slightly shorter exposure time and, in turn, a slightly higher per-pixel rms uncertainty. However, they yield a fainter point-source detection limit owing to the lower FWHM as measured in isolated and unsaturated stars. We choose to analyze the data separately from each night and only to combine the photometric measurements at the last step.

Random dithers of up to $40^{\prime \prime}$ are imposed between individual exposures to optimize the sky subtraction in the crowded cluster regions and to eliminate detector artifacts. Such large dithers require high point-source stability across the field. As a check, we estimate the pointing error at each dither position by stacking the object frames and then measuring the positional centroids of 13 pre-selected cluster members that span the full field of view and that are isolated from bright sources. We find that the typical translational shift between images is $\sim 2$ pixels, or $\sim 0$ ". 25 , with negligible rotation. The WCS information in each object frame is updated accordingly. At this point we resample the images onto the same pixel grid using the fluxconserved, overlapping pixel-area method in the Python routine astropy.reprojection, as needed.

We design our own reduction pipeline to ensure high flatness across the chip and to maximize the signal-to-noise ratio of the data. As a first step, we subtract the dark frames from all object frames. We then proceed to find the best estimate of the background. Within a single exposure, the sky background varies by $\sim 100$ ADUs, comparable to the integrated flux of some of the fainter cluster members. Therefore, instead of creating sky frames by taking the median at multiple pointings directly, we apply a "normalizing-rescaling" approach, in which we construct the master sky frames using exposures adjacent in time, which are then scaled to the background level of each object frame prior to the subtraction.

We designate each dither pointing " $i$ " as the co-addition of 24 individual object frames in $5 \mathrm{~s}$ exposures, all taken at the same position ( $120 \mathrm{~s}$ total science time), plus $\sim 0$ s readout time owing to nondestructive readouts. The $5 \mathrm{~s}$ exposure was chosen to be small to avoid persistence and nonlinearity effects. We find that a reasonable compromise in image combination is to 

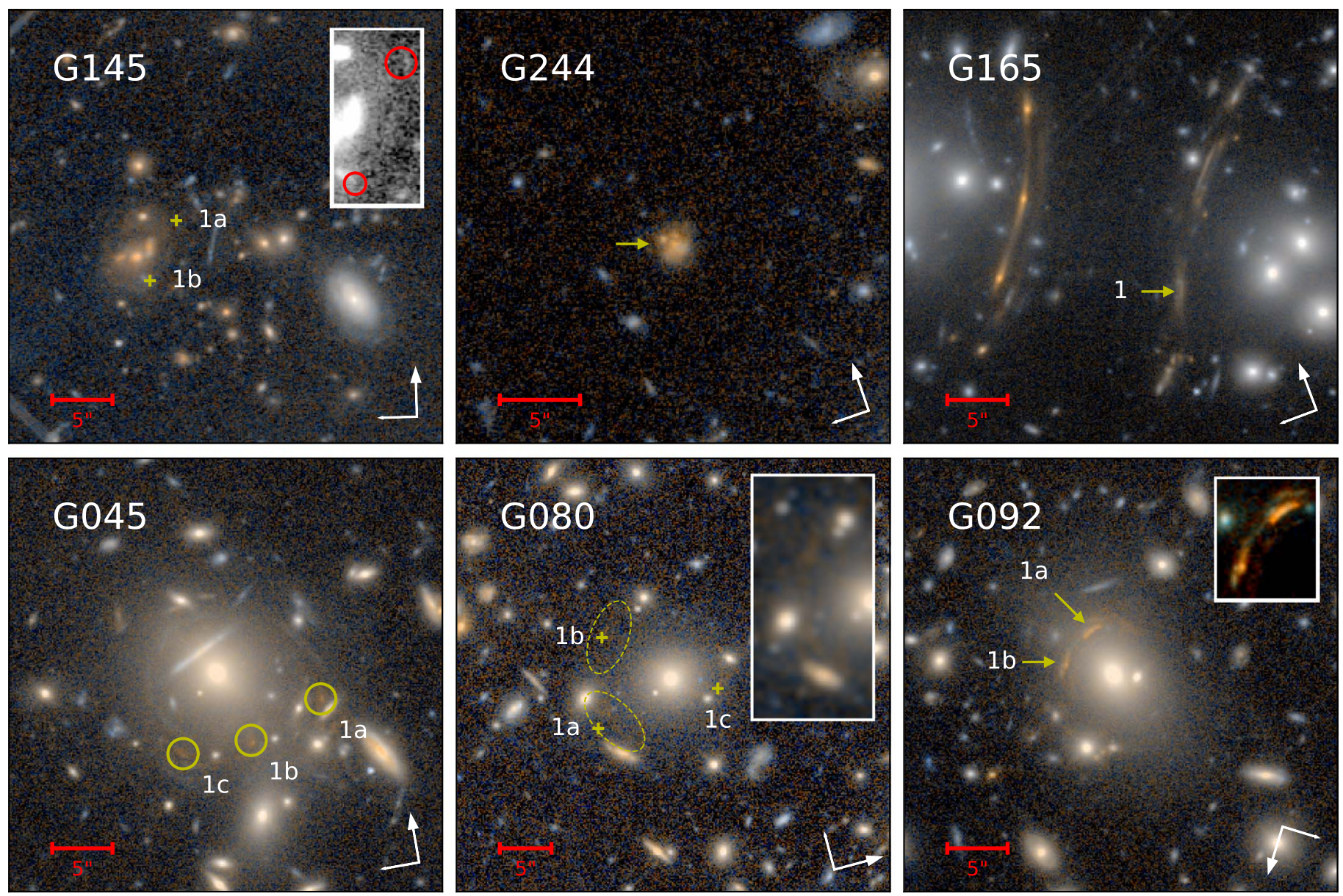

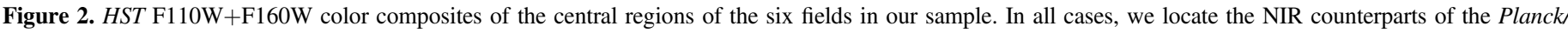

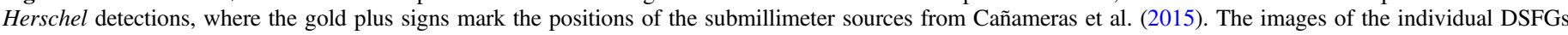

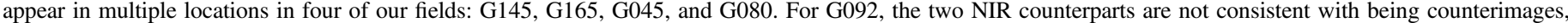

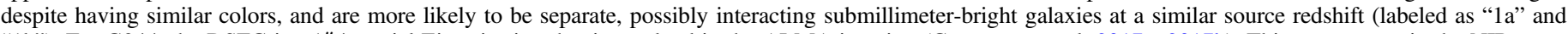

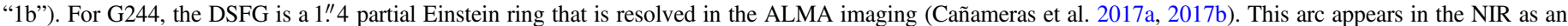

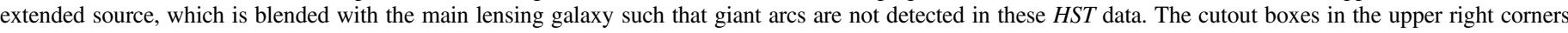

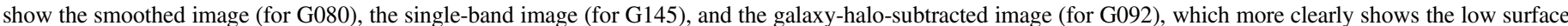

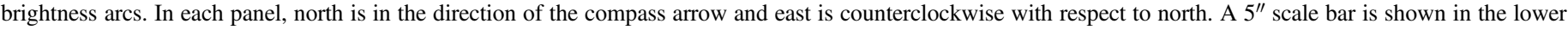
left corner of each panel for reference.

collect the temporally closest five dither pointings about each $i$ th dither pointing in a running boxcar, equating to a total clock time of $\sim 14$ minutes including overheads. The result of including more dither pointings is a slight improvement in the background noise but a degradation in image flatness. We mask out the bright sources in all the frames of the running boxcar to avoid biasing the result, or "master background," upward of its true value with unwanted cluster halo light. Before dividing this master frame into the $i$ th dither pointing, we divide the dither pointing frame by its five-pass, $3 \sigma$ clipped median value $^{18}$ to obtain the mean image of these "normalized" object frames. As a last step, we scale this new running master background frame to the $3 \sigma$ clipped median of this $i$ th dither pointing to match the sky-background level at the exact time of the exposure. Our background-subtracted dither pointings yield the "first-stage" result shown in Figure 3.

Following an iterative approach, we introduce two additional stages to the background subtraction, each time using the previous stage result as a starting point. The main difference is

\footnotetext{
18 When computing the $\sigma$-clipped statistics of an image, we cut the borders by 300 pixels to minimize the bias due to the hot/cold edges of infrared detectors.
}

that we continue to extend the bright object mask into the fainter outskirts of the masked sources. We avoid aggressively expanding the bright source mask, as increasing the number of masked pixels improves the flatness, but at the expense of the noise level, as fewer frames are available from which to estimate the background. The "third-stage" result is obtained by performing another iteration of the "second-stage" result. To assess image quality after each reduction stage, we compute the background rms values inside of seven test boxes of size $50 \times 200$ pixels located in regions isolated from bright sources. We find the background rms to decrease on average by $9 \%$ following the first stage and to converge to the $\lesssim 1 \%$ level following the second stage (see Figure 3).

To make corrections for pixel-to-pixel variations, we first tried applying a flat field to the data in the usual way prior to subtracting off the background. The result was unsatisfactory because image artifacts remained in the data. On reversing the order of these two operations, we found an improvement in the image flatness and the removal of the image artifacts. This improvement arises because our master flat field is constructed by combining local sky frames generated as a natural part of our background subtraction algorithm. Finally, we stacked the 
Table 2

The Sample of $H S T$ Lensed DSFGs

\begin{tabular}{|c|c|c|c|c|c|c|c|c|}
\hline \multirow[t]{2}{*}{ Arc ID } & \multirow{2}{*}{$\begin{array}{c}\text { R.A. } \\
(\mathrm{J} 2000)\end{array}$} & \multirow{2}{*}{$\begin{array}{c}\text { Decl. } \\
(\mathrm{J} 2000)\end{array}$} & \multirow{2}{*}{$\mathrm{F} 110 \mathrm{~W}_{\mathrm{AB}}$} & \multirow{2}{*}{$\mathrm{F} 160 \mathrm{~W}_{\mathrm{AB}}$} & \multirow{2}{*}{$\begin{array}{c}\text { Magnification } \\
(\mu)\end{array}$} & \multirow{2}{*}{$\begin{array}{l}\text { Lens Size }^{\mathrm{a}} \\
\quad(\operatorname{arcsec})\end{array}$} & \multicolumn{2}{|c|}{ Spectroscopic Redshift } \\
\hline & & & & & & & $z_{\text {lens }}$ & $z_{D S F G}$ \\
\hline$\overline{\text { G145_DSFG_1a }}$ & $10: 53: 22.250$ & $+60: 51: 48.95$ & $>26.9$ & $>26.2$ & $12 \pm 1$ & 5.9 & $0.837^{\mathrm{b}}$ & $3.6^{\mathrm{c}}$ \\
\hline G145_DSFG_1b & $10: 53: 22.563$ & $+60: 51: 44.03$ & $>26.9$ & $>26.2$ & $5 \pm 1$ & $\prime \prime$ & " & " \\
\hline G244_DSFG_1 & $10: 53: 53.107$ & $+05: 56: 18.44$ & $22.1_{-0.1}^{+0.1}$ & $21.0_{-0.1}^{+0.1}$ & $7-36^{\mathrm{d}}$ & $1.4^{\mathrm{d}}$ & $1.525^{\mathrm{d}}$ & $3.005^{\mathrm{d}}$ \\
\hline G165_DSFG_1a & $11: 27: 14.731$ & $+42: 28: 22.56$ & $23.0_{-0.1}^{+0.1}$ & $22.2_{-0.1}^{+0.1}$ & $\gtrsim 30$ & 13 & $0.351^{\mathrm{b}}$ & $2.2357^{\mathrm{e}}$ \\
\hline G165_DSFG_1b & $11: 27: 13.917$ & $+42: 28: 35.56$ & $>26.5$ & $>25.6$ & $\sim 8$ & $\prime \prime$ & $\prime \prime$ & $" \prime$ \\
\hline G045_DSFG_1a & $15: 02: 36.012$ & $+29: 20: 50.51$ & $>26.9$ & $25.5_{-0.2}^{+0.2}$ & $>9^{f}$ & 8 & $0.549^{c}$ & $3.427^{\mathrm{g}}$ \\
\hline G045_DSFG_1b & $15: 02: 36.479$ & $+29: 20: 47.90$ & $>27.0$ & $25.3_{-0.2}^{+0.2}$ & $>9^{f}$ & $\prime \prime$ & $\prime \prime$ & $\prime \prime$ \\
\hline G045_DSFG_1c & $15: 02: 36.921$ & $+29: 20: 47.96$ & $>26.7$ & $25.8_{-0.3}^{+0.3}$ & $>7^{\mathrm{f}}$ & $\prime \prime$ & $\prime \prime$ & $\prime \prime$ \\
\hline G080_DSFG_1a & $15: 44: 33.202$ & $+50: 23: 43.53$ & $>27.1$ & $>26.5$ & $\sim 20$ & 7 & $0.670^{c}$ & $2.6^{\mathrm{c}}$ \\
\hline G080_DSFG_1b & $15: 44: 32.483$ & $+50: 23: 41.69$ & $>27.5$ & $>26.5$ & $\sim 20$ & $\prime \prime$ & " & " \\
\hline G092_DSFG_1 $\mathrm{a}^{\mathrm{g}}$ & $16: 09: 17.842$ & $+60: 45: 19.41$ & $24.2_{-0.1}^{+0.1}$ & $23.0_{-0.1}^{+0.1}$ & $\sim 20$ & $\cdots$ & $0.45^{\mathrm{i}}$ & $3.256^{\mathrm{e}}$ \\
\hline G092_DSFG_1b ${ }^{g}$ & 16:09:17.693 & $+60: 45: 22.31$ & $25.0_{-0.2}^{+0.2}$ & $23.7_{-0.1}^{+0.1}$ & $\sim 20$ & $\cdots$ & " & " \\
\hline
\end{tabular}

Notes.

a The effective Einstein radius is reported, defined as $\theta_{E}=\sqrt{A / \pi}$, where $A$ is the area inside the critical curve (e.g., Acebron et al. 2018).

b The spectroscopic redshift comes from this paper.

c The spectroscopic redshift comes from Cañameras et al. (2015).

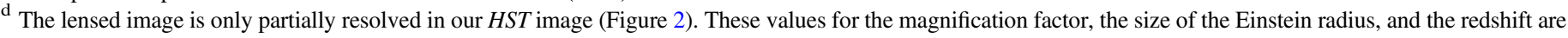
drawn from the ALMA data in Cañameras et al. (2017a, 2017b).

e The spectroscopic redshift comes from Harrington et al. (2016).

${ }^{\mathrm{f}}$ Magnification estimates for the three brightest emission-line regions of this arclet family were first reported in Nesvadba et al. (2016) as $\mu=10-22$.

g The spectroscopic redshift comes from Nesvadba et al. (2016).

${ }^{\mathrm{h}}$ We find that these two images are likely to originate from two different sources at a similar redshift.

${ }^{\mathrm{i}}$ The spectroscopic redshift comes from SDSS DR14 data.
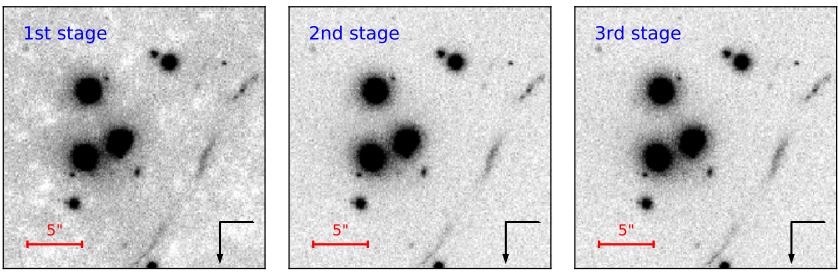

Figure 3. Background subtraction of our LBT/LUCI + ARGOS $K$-band imaging data is accomplished in an iterative process as illustrated above for a small region near the cluster center. The difference in image flatness between the stacked images after the first stage and the final image upon extending the bright object masks (second and third stages) is evident. We sample the background by placing test boxes down that are isolated from bright sources. We find the flatness to improve, or alternatively for the rms level of the background to decrease, by typically $9 \%$ between the first and second stages and to converge after the third stage. In each panel north is down and east is to the right.

sky-subtracted and flat-fielded object frames to produce our final data product. We report a mean $K$-band FWHM of 0 "' 53 for the 2016 December 9 run (LUCI2) and 0"' 29 for the 2016 December 15 run (LUCI1). In all, for the 2016 December 9 and 2016 December 15 runs, we reach a $10 \sigma$ limiting magnitude of $K_{\mathrm{AB}}=22.6 \mathrm{mag}$ and $K_{\mathrm{AB}}=23.5 \mathrm{mag}$ inside apertures of $4 \times$ FWHM, respectively. We do not combine the final images from the two different detectors, because the 2016 December 15 data have better spatial resolution (Figure 4). We emphasize, however, that our photometry is measured using both nights of data and weighted by an inverse-variance-weighted mean of the two fluxes, as described in Section 4.1.

The large aperture of LBT and the high resolution of LUCI + ARGOS have enabled rare detections of dozens of arcs in a ground-based image. Moreover, a side-by-side comparison of

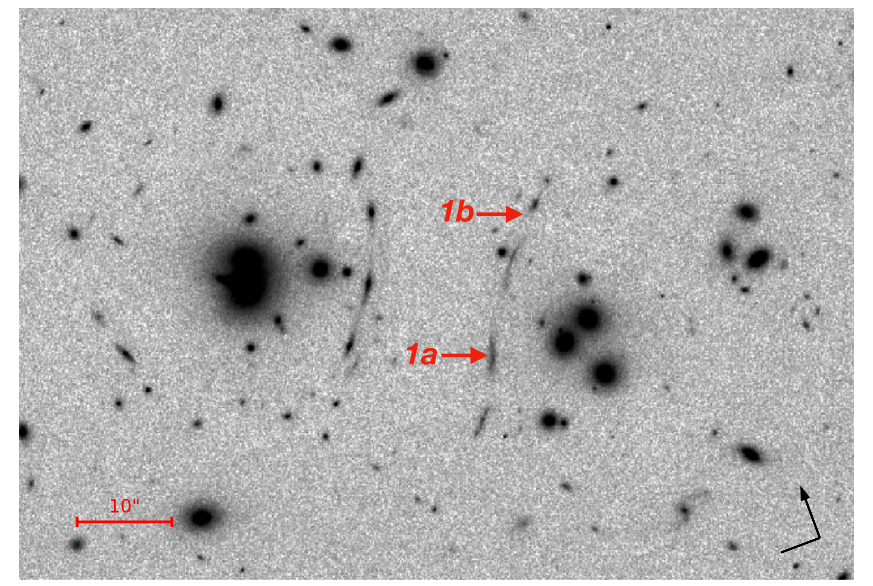

Figure 4. High-resolution $K$-band image $(\mathrm{FWHM} \approx 0$ !' 29$)$ for the central region of G165, using LBT/LUCI + ARGOS. Dozens of strongly lensed galaxy images and arclet families are detected. The lensed galaxy DSFG_1a is spatially resolved (labeled as "1a"). Notably, we identify a new image that we designate as its counterimage, DSFG_1b (labeled as " $1 \mathrm{~b}$ "). See Figure 5 for a three-band color image that includes these data alongside our HST data set. North is in the direction of the compass arrow, and east is to the left. A $10^{\prime \prime}$ scale bar is shown in the lower left corner for reference.

our $K$-band image and our HST WFC-IR images allows identifications of single galaxy images that appear in multiple positions in the image plane, or "arclet families." By this approach, we confirm the image position of the lensed DSFG, Arc $1 a$, and make the first detection of its counterimage, $A r c 1 b$, at its model-predicted location (Figure 5). Until now, we were unable to detect $A r c l b$ in our HST images, as the arc was too faint and too red. The $K$-band detection of this doubly imaged source with a known spectroscopic redshift is 

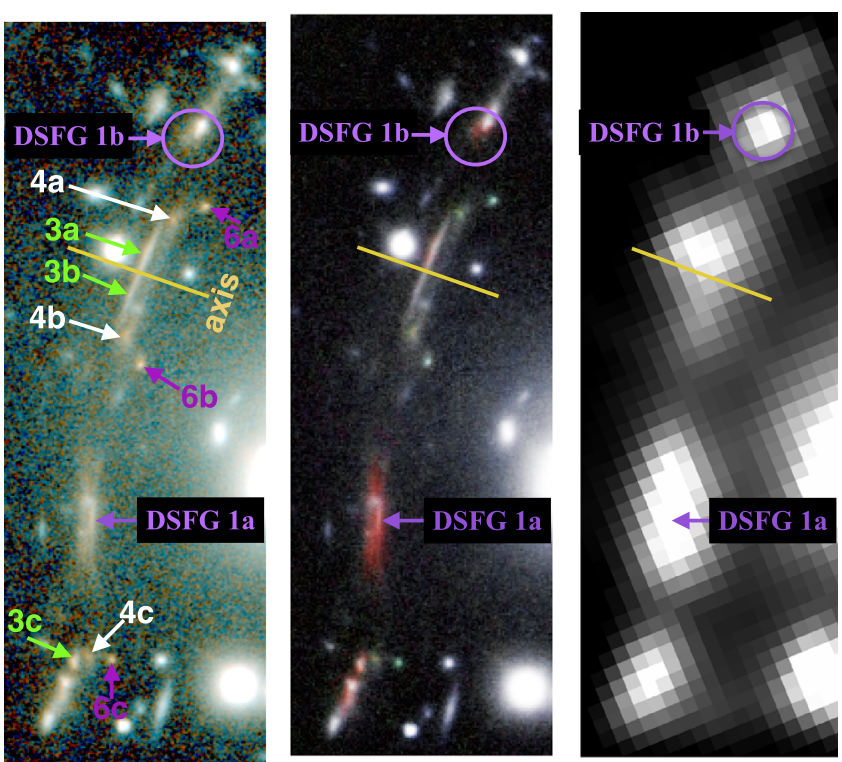

Figure 5. Left: two-band color image of a central region of the G165 field in HST WFC3-IR F110W+F160W. DSFG_1a appears at the expected location based on the submillimeter imaging (Cañameras et al. 2015). Sharing this crowded central region are three other arclet families, which all show fold images about an axis of symmetry, as labeled. Middle: three-band image of the HST WFC3-IR F110W (blue), F160W (green), and LBT/LUCI+ ARGOS $K$ (red) data. DSFG_1a stands out on account of its red color and is punctuated by two blue compact images, which may potentially be two images of compact star-forming regions arising from a single object in the background (see Section 6.2). Our lens model also predicts for there to be a fainter counterimage, DSFG_1b, which is discovered in the $K$-band data at its model-predicted location. Right: Spitzer/IRAC image in the $3.6 \mu \mathrm{m}$ band. DSFG_1a and DSFG_1b are both bright, with $S_{3.6, \mathrm{AB}} \approx 19.1 \mathrm{mag}$ and $S_{3.6, \mathrm{AB}} \sim 19.9 \mathrm{mag}$, respectively. All images are $10^{\prime \prime} \times 30^{\prime \prime}$ on a side and have the same orientation as in Figure 2.

impactful because it allows us to break the mass-sheet degeneracy to construct a robust mass map. In all, 11 arclet family members are detected in five separate arclet families. Thus, the LBT/LUCI+ARGOS $K$ imaging data effectively extend the wavelength reach of $H S T$, thereby opening up new discovery space that favors the redder galaxy populations. For additional details regarding the performance of the LBT/LUCI +ARGOS instrument, see Rabien et al. (2018).

\subsection{Spitzer Observations}

We acquired imaging in the G165 field on 2016 February 2 using the Spitzer InfraRed Camera (IRAC) in the $3.6 \mu \mathrm{m}$ and $4.5 \mu \mathrm{m}$ channels as part of a larger program (Cy13, GO-13024; PI: Yan) to image the fields of massive lensing clusters that would make good targets for JWST. The on-target exposure time was set to $12,000 \mathrm{~s}$ in each of the two channels. The Spitzer Science Center (SSC) processed these data using the standard SSC pipeline, and we made the final image mosaics based on these products. A detailed analysis of the data set of this entire program will appear in a separate paper $(\mathrm{H}$. Yan et al. 2018, in preparation). We also refer the reader to the description in Griffiths et al. (2018), where the reduction of their IRAC data from the same Spitzer program is discussed. In Figure 5, we show the $3.6 \mu \mathrm{m}$ IRAC mosaic of this field (right panel). The two images of the DSFG, G165_DSFG_la and $G 165 \_D S F G \_1 b$, are both bright, with $S_{3.6, \mathrm{AB}} \approx 19.1 \mathrm{mag}$ and $S_{3.6, \mathrm{AB}} \sim 19.9 \mathrm{mag}$, respectively.

\subsection{MMT Observations}

We obtained spectroscopy in the field of G165 on 2015 February 14 using MMT/Hectospec (Fabricant et al. 2013), as a part of a larger program (2015A; PI: Frye). Hectospec is a multifiber spectrograph that assigns optical fibers on the sky with minimum allowed separations of $\gtrsim 20^{\prime \prime}$. To maximize the wavelength range for measuring redshifts, we selected the 270 groove $\mathrm{mm}^{-1}$ grism, which covers a wavelength range of 3700-9150 $\AA$ at a dispersion of $1.21 \AA$ pixel $^{-1}$. We chose to position 23 fibers (20 galaxy targets plus 3 standard stars) with priorities set to the positions of the brighter examples of prominent giant arcs and cluster members, as selected by their NIR photometric redshift estimates made using CanadaFrance-Hawaii Telescope (CFHT) plus Spitzer/IRAC imaging. We refer to Cañameras et al. (2018) for details on the photometric analysis and photometric redshifts. Note that as the planning for this observing run took place prior to receiving the HST data set, we were not able to fine-tune the target list to include new arclet family members. The observations were composed of a single Hectospec pointing with $7 \times 1020 \mathrm{~s}$ exposures taken under variable seeing conditions of $\sim 1^{\prime \prime}-2^{\prime \prime}$. This was sufficient for our science goal given the 1".5 fiber widths and the relatively bright magnitudes of the targets of $i_{\mathrm{AB}} \simeq 18-22 \mathrm{mag}$.

The reductions proceeded in a standard fashion using the IDL/Hectospec Reduction Software package (HSRED) obtained from the Smithsonian Astrophysical Observatory Telescope Data Center. ${ }^{19}$ We removed cosmic rays using the code "LA Cosmic" (van Dokkum 2001). Corrections for pixelto-pixel variations, fringe corrections, and fiber identifications are accomplished using a dome flat. Background subtractions were made after first averaging together the spectra set to blank-sky positions, taken under the same conditions as the science data. The wavelength solution is found in two ways, using both an HeNeAr lamp exposure and the positions of prominent night-sky lines. We co-added the individual exposures to yield the final reduced spectra.

Secure spectroscopic measurements are made for 19 objects, which we define as the high-significance detection of two or more spectral features ( $>2 \sigma$ level in the continuum). Our catalog results in measurements for five new cluster members with $z=0.326-0.376$ and 11 new sources with redshifts $0.388<z<0.622$. Three galaxies have MMT/Hectospec redshifts that place them in the foreground. See Section 4.2 for additional details and the redshift catalog.

\subsection{Gemini Observations}

We obtained further spectroscopy in the field of G165 using the Gemini-North Multi-Object Spectrograph (GMOS) as a part of a larger program (GN-2016A-Q-30; PI: Frye). The observations took place on 2016 April 27. We selected the B600 line $\mathrm{mm}^{-1}$ grating, which has a wavelength coverage measured from our data of a total of $2975 \AA$ about the central wavelength for each slitlet at a dispersion of $0.92 \AA$ pixel $^{-1}$. As we did not have the HST images in time to plan this observing run, we populated the slit masks first with prominent arcs selected from the CFHT image from Cañameras et al. (2015), followed by cluster members selected from our Gemini preimaging data. We chose $1^{\prime \prime}$ slits to match typical seeing on-site.

\footnotetext{
${ }^{19}$ https://www.mmto.org/node/536
} 
We acquired six science exposures of $1200 \mathrm{~s}$, two each at central wavelengths of $645 \mathrm{~nm}, 650 \mathrm{~nm}$, and $655 \mathrm{~nm}$ to correct for chip gaps. Arc spectra were obtained within \pm 1 night of the observations using the CuAr lamps at similar central wavelengths. Dispersed flat-fields were taken at each of the three central wavelength (and hence grating tilt) configurations.

The initial calibrations of bias subtraction and flat-fielding proceeded in the standard way using the IRAF Gemini reduction package. ${ }^{20} \mathrm{We}$ removed cosmic rays prior to the background subtraction using the IRAF task GEMCRSPEC. For the wavelength calibration, there is a tendency for the IRAF algorithm to introduce wavelength offsets of the stacked spatial rows, especially for the smaller spectral "boxes." To avoid introducing this undesirable spatial feature into the data, we chose instead to use a pipeline written in IDL by one of us (B.L.F.). The IDL pipeline includes the tasks mentioned below and is discussed elsewhere (Frye et al. 2002, 2007, 2008). Briefly, the IDL pipeline avoids repixelization by identifying the flexure-induced instrumental curvature imprinted onto the individual spectrum box edges between the $2 \mathrm{D}$ spectra. This curvature amounts to 1-3 pixel shifts from the center to edge of the CCD, which are easily fit by low-order polynomials. We then wavelength-calibrate the data in two ways: using the arc lamps and using the night-sky lines. As both outputs had an rms on the wavelength fit of $<0.5 \AA$, we choose to use the sky lines for the potential benefit that the wavelength references are embedded directly onto the data at the time of the observations.

Cosmic-ray hits on the object were removed in 1D by a comparison of the stacked spectra from the six different exposures using our IDL task SPADD (Frye et al. 2002, 2007, 2008). Thresholds are set for the acceptable number of cosmic-ray hits per pixel in the stack to avoid removal of real spectral features. We measured redshifts for the 1D co-added spectra using our IDL task SPEC (Frye et al. 2002, 2007, 2008). Our catalog results in spectroscopic measurements for 32 galaxies in the G165 field. Of these, we find nine cluster members that are new with $0.326<z<0.376$ and 18 new lensed sources with $0.386<z<1.065$. Five galaxies have Gemini/GMOS redshifts that place them in the foreground of the lens. We refer to Section 4.2 for additional details and the full redshift catalog.

\section{Analysis and Results for G165}

We describe our algorithms for performing the matched photometry for the $H S T$ plus LBT imaging. We then analyze the combined results of the MMT, Gemini, and archival ground-based spectroscopy.

\subsection{The Photometry}

To include the LBT/LUCI + ARGOS data in our catalog alongside the $H S T$ photometry, we first translate the central locations of our photometric apertures defined by the F160W image onto the $K$ band using the WCS information. Although the FWHM resolution of our LUCI1 $K$-band data (FWHM $\sim 0$.' 29) is higher than that of our two HST bands (0." 22 and 0." 18 for F110W and F160W bands, respectively), we do not alter the aperture sizes and ellipticities, as there is

\footnotetext{
${ }^{20}$ IRAF is distributed by the National Optical Astronomy Observatories, which are operated by the Association of Universities for Research in Astronomy, Inc., under cooperative agreement with the National Science Foundation.
}

adequate matching to detect the vast majority of the sources. The data from LUCI1 and LUCI2 are obtained under different weather conditions, and the field orientation angles and plate scales are slightly different for LUCI1 and LUCI2. As a result, we opt to conduct $K$-band photometry separately for LUCI1 and LUCI2 images and only afterward to compute the aperture fluxes by applying an inverse-variance-weighted mean of the two values.

Table 3 gives the complete photometric catalog for all 11 arclet families. As the photometric depth at $K$ is shallower than for the $H S T J$ - and $H$-band data, the aperture fluxes for some sources and arclets fall below their $1 \sigma$ uncertainties. In such cases, we report the detection limit of the aperture fluxes. The redshift, $z_{\text {pred }}$, gives the predicted value for the redshift using our lens model. Notably, 11 arclet family members are detected in our LBT LUCI+ARGOS $K$-band image. They are Arcs $1 \mathrm{a}, 1 \mathrm{~b}$, Arcs 2a, 2b, 2c, Arcs 3a, 3b, 3c, Arcs 4a, 4b, 4c, and Arc 6b. The lensed DSFG, G165_DSFG_la, has F110W $\mathrm{W}_{\mathrm{AB}}=23.0_{-0.2}^{+0.2} \mathrm{mag}$, $\mathrm{F} 160 \mathrm{~W}_{\mathrm{AB}}=22.2_{-0.2}^{+0.2} \mathrm{mag}$, and $K_{\mathrm{AB}}=18.9_{-0.2}^{+0.2} \mathrm{mag}$, bright enough to make ground-based spectroscopic follow-up feasible.

\subsection{The Spectroscopy: G165 Cluster Members}

The catalog for all 62 objects in the G165 field with measured redshifts is given in Table 4 and presented as a redshift histogram in Figure 6. All objects in our redshift catalog are secure, by which we mean that we require that two or more spectroscopic features be detected at the $\gtrsim 2 \sigma$ level relative to the continuum. In the case of a single emission line, we require also the detection of a second significant feature such as a continuum break. We did not encounter any lone emission lines in this census. Hypothetically, if we did detect a single emission line blueward of $\mathrm{H} \alpha$ without a continuum break, then we would flag it as an Ly $\alpha$ candidate line. This is because a single emission line shortward of the rest-frame wavelength of $\mathrm{H} \alpha$ will likely be [O III] $\lambda \lambda 4959,5007$, [O II] $\lambda \lambda 3727,3729$, or Ly $\alpha$. In the first case, we would resolve both lines of the doublet. In the second case, the redshift would be sufficiently small, $z \lesssim 0.8$, that we would also detect [O III] $\lambda \lambda 4959,5007$ in our spectral bandpass for both the MMT/ Hectospec and Gemini/GMOS data sets. In the event that none of the above cases are encountered, then this would leave the likely identification of such a feature as $\operatorname{Ly} \alpha$. However, we do not detect any high-redshift candidates in this particular data set.

Typical absorption- and emission-line features identified in our data (depending on the redshift and type) are Fe II $\lambda \lambda 2587$, 2600, Mg II $\lambda \lambda 2796,2803, \mathrm{Mg}$ I $\lambda 2852$, [O II] $\lambda \lambda 3727$, 3729, $\mathrm{Ca} \mathrm{H}$ and $\mathrm{K}, G$ band, [O III] $\lambda \lambda 4959,5007, \mathrm{Mg}$ I $\lambda \lambda \lambda 5167,5173,5184, \mathrm{NaD}$, Balmer family $(\mathrm{H} \alpha$ through $\mathrm{H} \theta$ ), and [S II] $\lambda \lambda 6716,6731$. See Figure 7 for sample spectra. Note that all 50 secure redshifts obtained from our spectroscopy and reported in this paper with an " $\mathrm{H}$ " for Hectospec or "G" for Gemini/GMOS are new to the literature.

We specify cluster membership in the standard way, by requiring the redshifts to be in the range $0.326<z<0.376$, which corresponds to $\pm 3 \sigma$ with respect to the mean of $z=0.351$. In total, we have spectroscopic redshifts for 18 galaxies in the cluster. Of these, six cluster members are drawn from our MMT/ Hectospec data, and an additional nine cluster members come from our Gemini/GMOS data. The information for the remaining three cluster members comes from all other available sources, which for this field is only SDSS (DR14). Those cluster members 
Table 3

The PLCK_G165.7+67.0 (G165) Lensed DSFG and the Arclet Families

\begin{tabular}{|c|c|c|c|c|c|c|}
\hline $\begin{array}{l}\text { Arc } \\
\text { ID }\end{array}$ & $\begin{array}{l}\text { R.A. } \\
\text { (J2000) }\end{array}$ & $\begin{array}{l}\text { Decl. } \\
\text { (J2000) }\end{array}$ & $\begin{array}{r}\mathrm{F} 110 \mathrm{~W}_{\mathrm{AB}} \\
(\mathrm{mag})\end{array}$ & $\begin{array}{r}\mathrm{F} 160 \mathrm{~W}_{\mathrm{AB}} \\
(\mathrm{mag})\end{array}$ & $\begin{array}{r}K_{\mathrm{AB}} \\
(\mathrm{mag})\end{array}$ & $z_{\text {pred }}{ }^{a}$ \\
\hline G165_DSFG_1a & $11: 27: 14.731$ & $+42: 28: 22.56$ & $23.0_{-0.2}^{+0.2}$ & $22.2_{-0.2}^{+0.2}$ & $18.9_{-0.2}^{+0.2}$ & $2.2357^{\natural}$ \\
\hline G165_DSFG_1b & 11:27:13.917 & $+42: 28: 35.54$ & $>26.5$ & $>25.6$ & $22.6_{-0.2}^{+0.2}$ & 2.2357 \\
\hline G165_2a & $11: 27: 15.962$ & $+42: 28: 29.00$ & $22.8_{-0.1}^{+0.1}$ & $21.4_{-0.1}^{+0.1}$ & $18.5_{-0.1}^{+0.1}$ & 1.7 \\
\hline G165_2b & 11:27:15.606 & $+42: 28: 34.18$ & $22.8_{-0.1}^{+0.1}$ & $21.3_{-0.1}^{+0.1}$ & $18.3_{-0.1}^{+0.1}$ & $" \prime$ \\
\hline G165_2c & $11: 27: 15.325$ & $+42: 28: 41.32$ & $22.8_{-0.1}^{+0.1}$ & $21.4_{-0.1}^{+0.1}$ & $18.5_{-0.1}^{+0.1}$ & " \\
\hline G165_3a & $11: 27: 14.146$ & $+42: 28: 32.00$ & $25.0_{-0.1}^{+0.1}$ & $24.3_{-0.1}^{+0.1}$ & $21.6_{-0.1}^{+0.1}$ & 2.7 \\
\hline G165_3b & $11: 27: 14.330$ & $+42: 28: 30.36$ & $25.6_{-0.2}^{+0.2}$ & $25.1_{-0.2}^{+0.2}$ & $22.3_{-0.2}^{+0.3}$ & $"$ \\
\hline G165_3c & 11:27:14.969 & $+42: 28: 17.34$ & $25.3_{-0.2}^{+0.2}$ & $24.6_{-0.2}^{+0.2}$ & $21.9_{-0.3}^{+0.3}$ & " \\
\hline G165_4a & $11: 27: 14.059$ & $+42: 28: 32.73$ & $26.1_{-0.2}^{+0.3}$ & $24.8_{-0.1}^{+0.1}$ & $22.7_{-0.3}^{+0.4}$ & 2.7 \\
\hline G165_4b & $11: 27: 14.372$ & $+42: 28: 29.00$ & $25.8_{-0.2}^{+0.2}$ & $24.7_{-0.2}^{+0.2}$ & $22.2_{-0.2}^{+0.2}$ & $\prime \prime$ \\
\hline G165_4c & 11:27:14.909 & $+42: 28: 17.33$ & $>26.9$ & $26.0_{-0.2}^{+0.2}$ & $>23.6$ & $" 1$ \\
\hline G165_5a & $11: 27: 13.187$ & $+42: 28: 25.83$ & $25.8_{-0.2}^{+0.2}$ & $25.7_{-0.2}^{+0.3}$ & $>23.0$ & 4.3 \\
\hline G165_5b & $11: 27: 13.188$ & $+42: 28: 24.67$ & $25.7_{-0.2}^{+0.2}$ & $25.5_{-0.2}^{+0.2}$ & $>23.4$ & $" 1$ \\
\hline G165_6a & $11: 27: 13.924$ & $+42: 28: 32.79$ & $25.8_{-0.2}^{+0.2}$ & $24.7_{-0.1}^{+0.1}$ & $>23.2$ & 2.7 \\
\hline G165_6b & $11: 27: 14.358$ & $+42: 28: 27.72$ & $25.8_{-0.2}^{+0.2}$ & $24.5_{-0.1}^{+0.1}$ & $23.2_{-0.4}^{+0.7}$ & $" \prime$ \\
\hline G165_6c & $11: 27: 14.836$ & $+42: 28: 16.88$ & $>26.3$ & $25.1_{-0.2}^{+0.2}$ & $>23.2$ & $" 1$ \\
\hline G165_7a & $11: 27: 15.300$ & $+42: 28: 38.35$ & $>26.2$ & $25.6_{-0.3}^{+0.4}$ & $>23.5$ & 1.7 \\
\hline G165_7b & $11: 27: 15.397$ & $+42: 28: 35.96$ & $>26.9$ & $25.2_{-0.2}^{+0.3}$ & $>23.5$ & $"$ \\
\hline G165_7c & $11: 27: 16.083$ & $+42: 28: 25.84$ & $26.2_{-0.3}^{+0.4}$ & $25.7_{-0.4}^{+0.5}$ & $>23.5$ & $"$ \\
\hline G165_8a & $11: 27: 15.210$ & $+42: 28: 40.85$ & $>26.0$ & $>25.3$ & $>23.0$ & 1.7 \\
\hline G165_8b & $11: 27: 15.581$ & $+42: 28: 32.37$ & $>26.3$ & $>25.5$ & $>23.0$ & $"$ \\
\hline G165_8c & $11: 27: 15.834$ & $+42: 28: 28.36$ & $>26.8$ & $>26.2$ & $>23.0$ & " \\
\hline G165_9a & $11: 27: 15.423$ & $+42: 28: 40.59$ & $26.5_{-0.3}^{+0.4}$ & $>25.6$ & $>22.9$ & 1.5 \\
\hline G165_9b & $11: 27: 15.568$ & $+42: 28: 36.06$ & $>26.4$ & $>25.6$ & $>22.4$ & $"$ \\
\hline G165_9c & $11: 27: 16.096$ & $+42: 28: 28.03$ & $>26.6$ & $>25.6$ & $>22.7$ & $"$ \\
\hline G165_10a & $11: 27: 15.171$ & $+42: 28: 38.92$ & $>26.3$ & $26.2_{-0.3}^{+0.5}$ & $>22.8$ & 1.7 \\
\hline G165_10b & $11: 27: 15.453$ & $+42: 28: 32.90$ & $26.5_{-0.3}^{+0.3}$ & $25.9_{-0.3}^{+0.3}$ & $>23.0$ & " \\
\hline G165_11a & $11: 27: 15.761$ & $+42: 28: 42.28$ & $24.7_{-0.1}^{+0.1}$ & $24.5_{-0.1}^{+0.1}$ & $>22.0$ & 2.1 \\
\hline G165_11b & $11: 27: 15.784$ & $+42: 28: 40.45$ & $25.0_{-0.1}^{+0.1}$ & $24.8_{-0.2}^{+0.2}$ & $>22.0$ & $"$ \\
\hline G165_11c & $11: 27: 16.510$ & $+42: 28: 26.12$ & $25.0_{-0.1}^{+0.1}$ & $24.7_{-0.1}^{+0.1}$ & $>22.7$ & " \\
\hline
\end{tabular}

Notes.

${ }^{a}$ Unless otherwise noted, these redshifts are predictions drawn from our strong-lensing model that await spectroscopic confirmation.

b The spectroscopic redshift for G165_DSFG_1a comes from Harrington et al. (2016).

in common with the smaller HST field fall reasonably well onto the red sequence of the color-magnitude diagram (CMD; see Section B.1). Five galaxies with spectroscopic redshifts extending behind the cluster and in the range $0.412 \leqslant z \leqslant 0.414$ are not included in this cluster member set, yet they may be members of an unrelated background galaxy group (Figure 6, inset).

To cope with the small sample size, we choose to measure the velocity dispersion using the Gapper method (see Hou et al. 2009 for details). We compute a velocity dispersion of $\sigma=2400 \pm 620 \mathrm{~km} \mathrm{~s}^{-1}$ from the 18 cluster members, corresponding to a large value for the virial mass of $M_{V}=(9.1 \pm 0.4) \times 10^{15} M_{\odot}$ within $1 \mathrm{Mpc}$. If we now restrict the angular extent to match the scale of our HST observations of $\theta=50^{\prime \prime}$, or $\approx 250 \mathrm{kpc}$, then 13 cluster members are removed. The velocity dispersion for this smaller redshift set is $\sigma=2000 \pm$ $300 \mathrm{~km} \mathrm{~s}^{-1}$, yielding again a large value for the mass of $M_{V}=1.3_{-0.70}^{+0.04} \times 10^{15} M_{\odot}$. The uncertainties on the velocity dispersion are found by summing up the uncertainties in the galaxy redshifts in quadrature. The value for the dynamical mass within the cluster core is not uncommon for massive clusters (Girardi et al. 1993), and at the same time it is higher than the mean value for CLASH clusters by a factor of three (Siegel et al. 2016).
We emphasize that our values for $\sigma$, and hence also for $M_{V}$, will be biased upward relative to the true value if the line-ofsight velocities are enhanced relative to those in the transverse direction. It is relevant here to consider a nonspherical velocity structure, as a bimodal mass distribution is evident in the imaging data. The cluster galaxies separate out naturally into two main mass concentrations: a northeast (NE) and a southwest (SW) region. We take the cluster center to be situated at the center of this bimodal mass distribution, with a positional uncertainty that depends on the relative masses. Given that the two mass regions produce similar numbers of arcs and arclet families, conservatively we expect the mass ratio to be $\lesssim 10$. The uncertainty on the cluster center translates into an uncertainty in the virial radius, and none of the above takes into account the potentially large systemic errors due to the unknown true radial and velocity structure of the cluster. We return to the discussion of the cluster kinematics in Section 6.1.

\section{Strong-lens Modeling}

\subsection{The Approach}

We perform a strong-lensing analysis for the fields in our sample by an approach that relies on the assumption that the 
Table 4

Spectroscopic Redshifts in the G165 Field ${ }^{\mathrm{a}}$

\begin{tabular}{|c|c|c|c|c|c|c|c|}
\hline Source ID & $\begin{array}{c}\text { R.A. } \\
(\mathrm{J} 2000)\end{array}$ & $\begin{array}{c}\text { Decl. } \\
(\mathrm{J} 2000)\end{array}$ & $z_{\text {spec }}$ & Ref & $\begin{array}{c}i_{\mathrm{SDSS}, \mathrm{AB}} \\
\quad(\mathrm{mag})\end{array}$ & $\begin{array}{c}\mathrm{F} 110 \mathrm{~W}_{\mathrm{AB}} \\
(\mathrm{mag})\end{array}$ & $\begin{array}{c}\mathrm{F} 160 \mathrm{~W}_{\mathrm{AB}} \\
\quad(\mathrm{mag})\end{array}$ \\
\hline s1 & $11: 26: 36.173$ & $+42: 30: 08.42$ & 0.621 & $\mathrm{~S}$ & 18.99 & $\ldots$ & $\ldots$ \\
\hline s2 & $11: 26: 43.968$ & $+42: 31: 05.16$ & 0.274 & $\mathrm{~S}$ & 18.17 & $\ldots$ & $\cdots$ \\
\hline s3 & $11: 26: 45.732$ & $+42: 28: 15.65$ & 0.121 & $\mathrm{~S}$ & 17.05 & $\ldots$ & $\cdots$ \\
\hline s4 & $11: 26: 46.387$ & $+42: 26: 51.81$ & 0.412 & $\mathrm{~S}$ & 18.76 & $\cdots$ & $\cdots$ \\
\hline s5 & $11: 26: 48.850$ & $+42: 28: 33.05$ & 0.471 & $\mathrm{~S}$ & 19.59 & $\cdots$ & $\cdots$ \\
\hline s6 & $11: 26: 59.151$ & $+42: 30: 11.10$ & 0.412 & $\mathrm{H}$ & 21.34 & $\ldots$ & $\ldots$ \\
\hline s7 & $11: 26: 59.471$ & $+42: 28: 10.81$ & 0.346 & $\mathrm{H}$ & 18.74 & $\ldots$ & $\cdots$ \\
\hline s8 & $11: 26: 59.999$ & $+42: 27: 03.71$ & 0.388 & $\mathrm{H}$ & 19.77 & $\ldots$ & $\cdots$ \\
\hline s9 & $11: 27: 00.233$ & $+42: 31: 03.06$ & 0.232 & $\mathrm{H}$ & 17.84 & $\ldots$ & $\ldots$ \\
\hline s10 & $11: 27: 02.557$ & $+42: 29: 12.35$ & 0.445 & $\mathrm{H}$ & 19.68 & $\ldots$ & $\ldots$ \\
\hline s11 & $11: 27: 02.667$ & $+42: 27: 20.19$ & 0.348 & $\mathrm{G}$ & 20.02 & $\ldots$ & $\cdots$ \\
\hline $\mathrm{s} 12$ & $11: 27: 04.239$ & $+42: 29: 32.38$ & 0.399 & $\mathrm{G}$ & 21.46 & $\ldots$ & $\cdots$ \\
\hline s13 & $11: 27: 04.785$ & $+42: 31: 21.53$ & 0.389 & $\mathrm{H}$ & 20.15 & $\cdots$ & $\cdots$ \\
\hline s14 & $11: 27: 05.558$ & $+42: 25: 55.06$ & 0.445 & $\mathrm{G}$ & 21.42 & $\ldots$ & $\cdots$ \\
\hline s15 & $11: 27: 05.754$ & $+42: 27: 34.60$ & 0.623 & $\mathrm{G}$ & $\ldots$ & $\ldots$ & $\cdots$ \\
\hline s16 & $11: 27: 06.732$ & $+42: 27: 50.40$ & 0.275 & $\mathrm{G}$ & 17.50 & $\ldots$ & $\cdots$ \\
\hline s17 & $11: 27: 06.787$ & $+42: 27: 23.25$ & 0.623 & $\mathrm{G}$ & $\ldots$ & $\ldots$ & $\cdots$ \\
\hline s18 & $11: 27: 07.552$ & $+42: 28: 22.50$ & 0.622 & $\mathrm{H}$ & 20.35 & $\ldots$ & $\ldots$ \\
\hline s19 & 11:27:09.420 & $+42: 30: 38.23$ & 0.624 & $\mathrm{G}$ & 21.01 & $\ldots$ & $\cdots$ \\
\hline s20 & $11: 27: 09.564$ & $+42: 30: 10.90$ & 0.358 & $\mathrm{G}$ & $\cdots$ & $\ldots$ & $\ldots$ \\
\hline s21 & $11: 27: 10.774$ & $+42: 30: 14.15$ & 0.033 & $\mathrm{~S}$ & 14.71 & $\ldots$ & $\cdots$ \\
\hline $\mathrm{s} 22$ & 11:27:11.137 & $+42: 26: 50.88$ & 0.412 & $\mathrm{H}$ & 18.56 & $\cdots$ & $\cdots$ \\
\hline s23 & $11: 27: 12.283$ & $+42: 28: 23.88$ & 0.353 & $\mathrm{H}$ & 21.08 & 20.2 & 19.9 \\
\hline s24 & $11: 27: 13.046$ & $+42: 27: 09.58$ & 0.386 & $\mathrm{G}$ & 21.25 & 21.4 & 21.3 \\
\hline s25 & $11: 27: 13.133$ & $+42: 31: 09.47$ & 0.510 & $\mathrm{~S}$ & 19.29 & $\cdots$ & $\cdots$ \\
\hline s26 & $11: 27: 13.300$ & $+42: 30: 27.68$ & 0.347 & $\mathrm{G}$ & 21.30 & $\ldots$ & $\ldots$ \\
\hline s27 & $11: 27: 13.444$ & $+42: 27: 00.54$ & 0.411 & $\mathrm{G}$ & 20.46 & $\cdots$ & $\cdots$ \\
\hline s28 & $11: 27: 13.653$ & $+42: 30: 39.21$ & 0.374 & $\mathrm{G}$ & 21.29 & $\ldots$ & $\cdots$ \\
\hline s29 & $11: 27: 13.680$ & $+42: 28: 22.44$ & 0.348 & $\mathrm{~S}$ & 18.33 & 18.7 & 18.3 \\
\hline s30 & $11: 27: 14.803$ & $+42: 27: 37.58$ & 0.135 & $\mathrm{H}$ & 19.06 & 18.6 & 18.2 \\
\hline s31 & $11: 27: 15.312$ & $+42: 29: 00.99$ & 0.305 & $\mathrm{G}$ & 20.18 & 22.0 & 21.7 \\
\hline $\mathrm{s} 32$ & $11: 27: 15.370$ & $+42: 27: 35.60$ & 1.065 & $\mathrm{G}$ & 19.06 & 22.0 & 21.9 \\
\hline s33 & $11: 27: 16.596$ & $+42: 28: 40.99$ & 0.348 & $\mathrm{~S}$ & 18.00 & 18.3 & 17.9 \\
\hline s34 & $11: 27: 16.664$ & $+42: 27: 23.07$ & 0.720 & $\mathrm{G}$ & 22.24 & $\ldots$ & $\cdots$ \\
\hline s35 & $11: 27: 16.692$ & $+42: 28: 38.15$ & 0.338 & $\mathrm{~S}$ & 17.16 & $\ldots$ & $\cdots$ \\
\hline s36 & $11: 27: 16.894$ & $+42: 31: 08.83$ & 0.508 & $\mathrm{G}$ & 21.35 & $\cdots$ & $\cdots$ \\
\hline s37 & $11: 27: 17.145$ & $+42: 26: 07.18$ & 0.146 & $\mathrm{G}$ & 21.62 & $\ldots$ & $\cdots$ \\
\hline s38 & $11: 27: 17.928$ & $+42: 27: 20.43$ & 0.193 & $\mathrm{H}$ & 18.41 & $\ldots$ & $\cdots$ \\
\hline s39 & $11: 27: 18.027$ & $+42: 26: 48.30$ & 0.368 & $\mathrm{G}$ & 20.23 & $\ldots$ & $\cdots$ \\
\hline $\mathrm{s} 40$ & $11: 27: 18.501$ & $+42: 26: 02.94$ & 0.623 & $\mathrm{G}$ & $\cdots$ & $\cdots$ & $\cdots$ \\
\hline s41 & $11: 27: 18.594$ & $+42: 29: 29.25$ & 0.471 & $\mathrm{G}$ & $\cdots$ & 22.9 & 22.7 \\
\hline $\mathrm{s} 42$ & $11: 27: 18.652$ & $+42: 28: 09.81$ & 0.354 & $\mathrm{G}$ & $\cdots$ & 21.6 & 21.3 \\
\hline $\mathrm{s} 43$ & $11: 27: 18.879$ & $+42: 29: 55.38$ & 0.254 & $\mathrm{G}$ & 21.44 & $\ldots$ & $\cdots$ \\
\hline s44 & $11: 27: 19.394$ & $+42: 29: 50.95$ & 0.346 & $\mathrm{H}$ & 20.28 & $\ldots$ & $\cdots$ \\
\hline s45 & $11: 27: 19.452$ & $+42: 27: 01.73$ & 0.723 & $\mathrm{G}$ & - & $\cdots$ & $\cdots$ \\
\hline s46 & $11: 27: 19.908$ & $+42: 30: 18.18$ & 0.351 & $\mathrm{G}$ & 20.59 & $\ldots$ & $\ldots$ \\
\hline s47 & $11: 27: 20.146$ & $+42: 29: 18.46$ & 0.275 & $\mathrm{G}$ & 20.40 & 24.2 & 24.0 \\
\hline s 48 & $11: 27: 20.379$ & $+42: 30: 28.11$ & 0.999 & $\mathrm{G}$ & 20.97 & $\cdots$ & $\cdots$ \\
\hline s49 & $11: 27: 20.386$ & $+42: 30: 51.64$ & 0.443 & $\mathrm{H}$ & $\cdots$ & $\cdots$ & $\cdots$ \\
\hline s50 & $11: 27: 20.458$ & $+42: 27: 59.16$ & 0.345 & $\mathrm{H}$ & 19.17 & 18.7 & 18.4 \\
\hline s51 & $11: 27: 20.509$ & $+42: 29: 01.78$ & 0.414 & $\mathrm{G}$ & - & $\cdots$ & $\cdots$ \\
\hline s52 & $11: 27: 22.652$ & $+42: 31: 08.80$ & 0.344 & $\mathrm{H}$ & 19.54 & $\ldots$ & $\cdots$ \\
\hline s53 & $11: 27: 23.369$ & $+42: 29: 53.01$ & 0.348 & $\mathrm{G}$ & 21.04 & $\ldots$ & $\cdots$ \\
\hline s54 & $11: 27: 23.383$ & $+42: 26: 27.56$ & 0.914 & $\mathrm{G}$ & 20.12 & $\ldots$ & $\cdots$ \\
\hline s55 & $11: 27: 23.685$ & $+42: 26: 49.52$ & 0.916 & $\mathrm{G}$ & $\ldots$ & $\ldots$ & $\ldots$ \\
\hline s56 & $11: 27: 23.833$ & $+42: 28: 42.64$ & $0.0^{b}$ & $\mathrm{H}$ & 18.51 & $\ldots$ & $\cdots$ \\
\hline s57 & $11: 27: 24.564$ & $+42: 29: 48.99$ & 0.347 & $\mathrm{G}$ & 21.06 & $\ldots$ & $\cdots$ \\
\hline s58 & $11: 27: 24.695$ & $+42: 29: 04.92$ & 0.759 & $\mathrm{G}$ & 21.72 & $\ldots$ & $\cdots$ \\
\hline s59 & $11: 27: 25.340$ & $+42: 27: 43.93$ & 0.395 & $\mathrm{H}$ & 22.12 & $\cdots$ & $\cdots$ \\
\hline s60 & $11: 27: 26.521$ & $+42: 26: 58.02$ & 0.347 & $\mathrm{H}$ & 19.55 & $\ldots$ & $\cdots$ \\
\hline s61 & $11: 27: 29.150$ & $+42: 30: 23.45$ & 0.544 & $\mathrm{~S}$ & 19.36 & $\cdots$ & $\cdots$ \\
\hline s62 & $11: 27: 31.872$ & $+42: 27: 41.04$ & 0.522 & $\mathrm{~S}$ & 19.60 & $\ldots$ & $\cdots$ \\
\hline
\end{tabular}

Note.

${ }^{\mathrm{a}}$ This object is a star. 


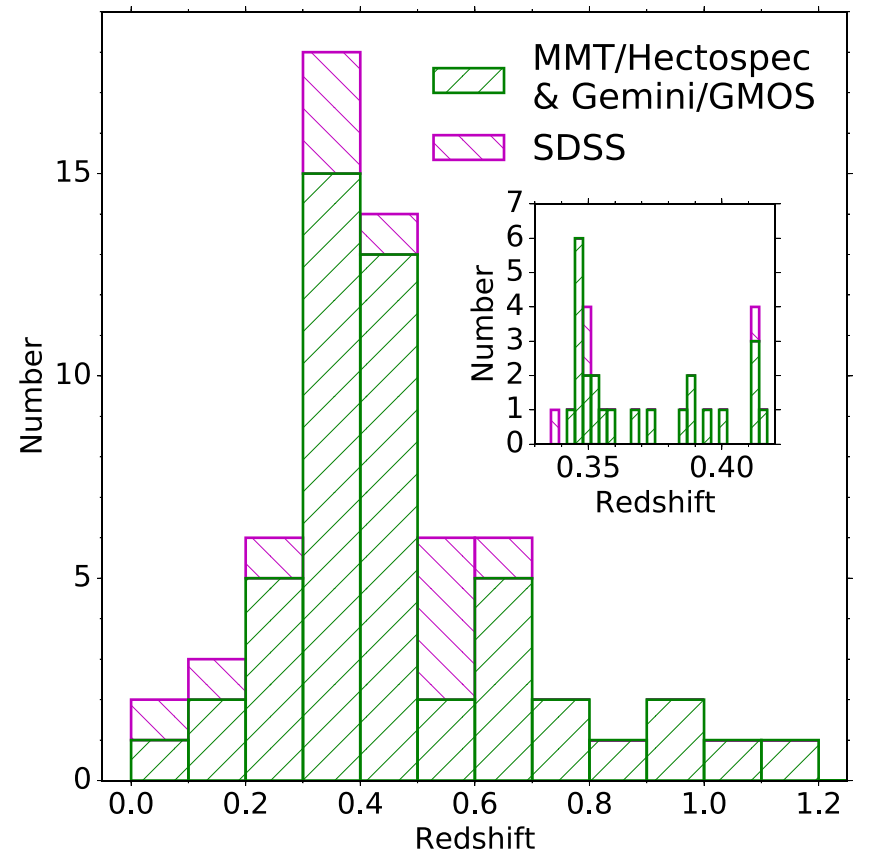

Figure 6. Histogram of spectroscopic redshifts in the G165 field. The redshift catalog combines results from our MMT/Hectospec and Gemini/GMOS data sets (green) and objects drawn from the literature (SDSS, DR14; magenta). We measure a value for the lens redshift of $z=0.351$, based on 18 cluster members in the range of $0.326<z<0.376$. The inset histogram peaks at the lens redshift and shows a secondary peak at higher redshift that may indicate the presence of an unrelated background galaxy group. These cluster members range in position from the center out to a cluster-centric radius of $\approx 0.8 \mathrm{Mpc}$.

light approximately traces the mass, or "LTM," such that the galaxies are biased tracers of the dark matter. A similar LTM methodology has been used to constrain the 2D mass distribution for cluster lenses extending back to some of the first examples of image multiplicities in cluster environments, such as A2390 (Frye \& Broadhurst 1998) and C10024 (Broadhurst et al. 2000). This lensing analysis was subsequently extended to accommodate the properties of the first cluster field to show large numbers of arclet families, namely, the HST Advanced Camera for Surveys (ACS) image of A1689 (Broadhurst et al. 2005). To construct our mass maps, we use the well-tested implementation of the LTM pipeline by Zitrin et al. (2009, 2015). We also refer to Acebron et al. (2018) and Cibirka et al. (2018) for additional descriptions.

In the LTM model, the lensing galaxies are assigned a power-law mass density distribution scaled in proportion to their luminosities. The power-law index is left as a free parameter and is the same for all lensing galaxies. The superposition of the mass distributions of the individual lensing galaxies, which makes up the initial 2D mass distribution, is then smoothed by a Gaussian kernel to approximate the dark matter distribution, whose width is the second free parameter of the model. The dominant dark matter and galaxy distributions are, in turn, summed up with a relative weight, which adds another free parameter of the model, and then they are normalized (to a specific source redshift), which necessitates the fourth free parameter. Finally, the model accommodates a two-parameter external shear to provide additional flexibility. The values for these six parameters are constrained by the positions, orientations, and relative brightnesses of the arclet families. The best-fit model and errors are optimized through a Monte Carlo Markov Chain using thousands of steps.

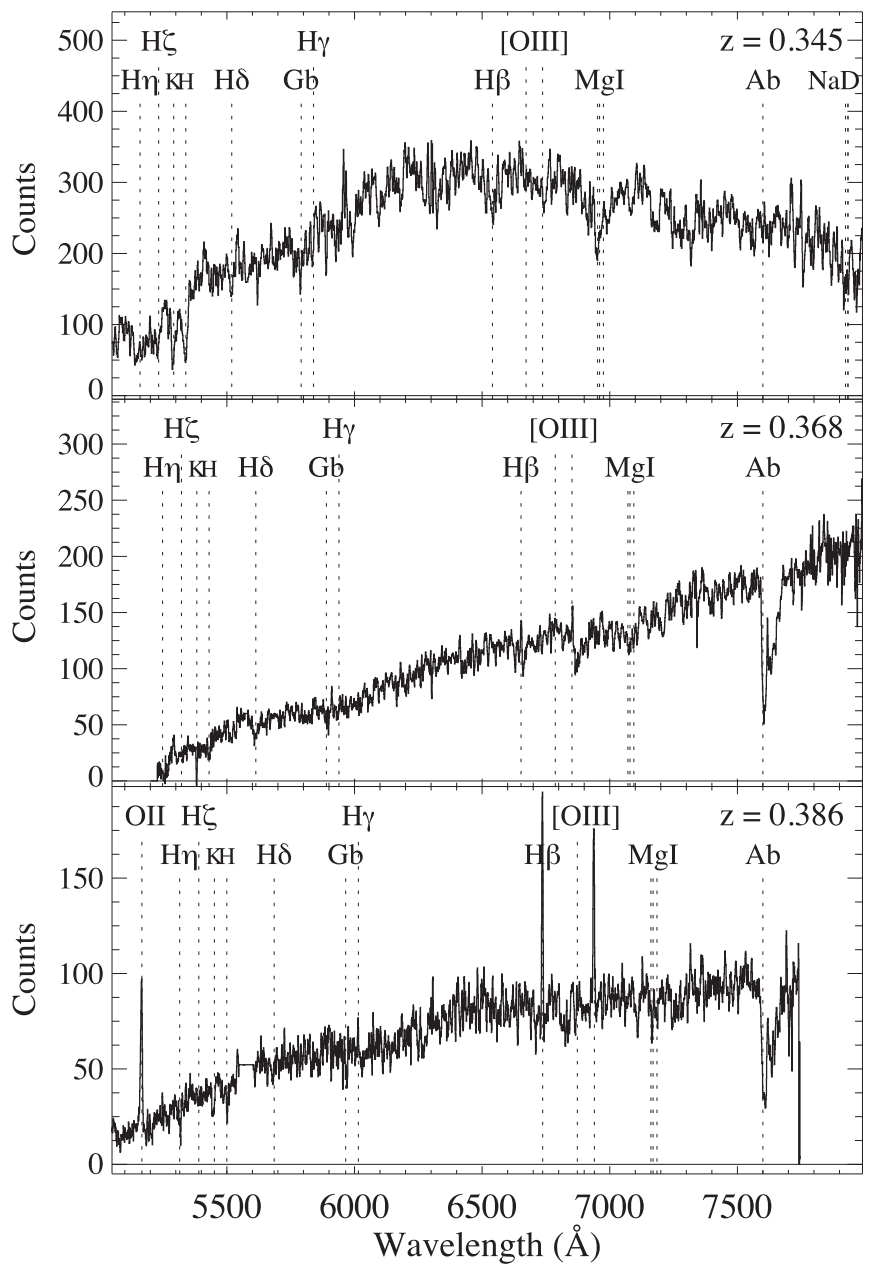

Figure 7. Left: sample spectra of cluster members in the field of G165. Only spectroscopy that yields secure redshifts are used in this study. The galaxies typically show the absorption features expected of early-type galaxies such as the MMT/Hectospec spectrum of Source ID "s50" (top panel). Other objects show a combination of stellar absorption plus emission line features, such as the Gemini/GMOS spectrum of Source ID "s39" (middle panel). Three cluster members show nebular emission line features, such as the Gemini/GMOS spectrum of Source ID "s24" (bottom panel). See Table 4 for details.

The exquisite spatial resolution of HST makes feasible the designation of arclet families based on morphology and color. Moreover, the HST images show obvious axes of symmetry superimposed onto the field (see example in Figure 5), which allow for the identification of image multiplicities even without the aid of measured redshifts in some cases. Arclet families, in turn, constrain the model by imposing the condition that each family member image originates from the same source. The best-fit model is the one that minimizes the angular separations between the observed and predicted (relensed) image positions in the image plane. Notably, in addition to providing confirmation of the locations of the counterimages, the strong-lensing model also has predictive power to locate new image counterparts that can be searched for in the data to iteratively improve on the model result. Because secure spectroscopic redshifts are not available for every arclet family, the ratios of the relative angular diameter distances of the lens to the source, $d_{L S}$, and of the observer to the source, $d_{S}$, are left as free parameters to be optimized in the minimization of the model. In such cases, we allow a wide range of relative values of $d_{L S} / d_{S}= \pm 0.12$, which equates roughly to a redshift range 


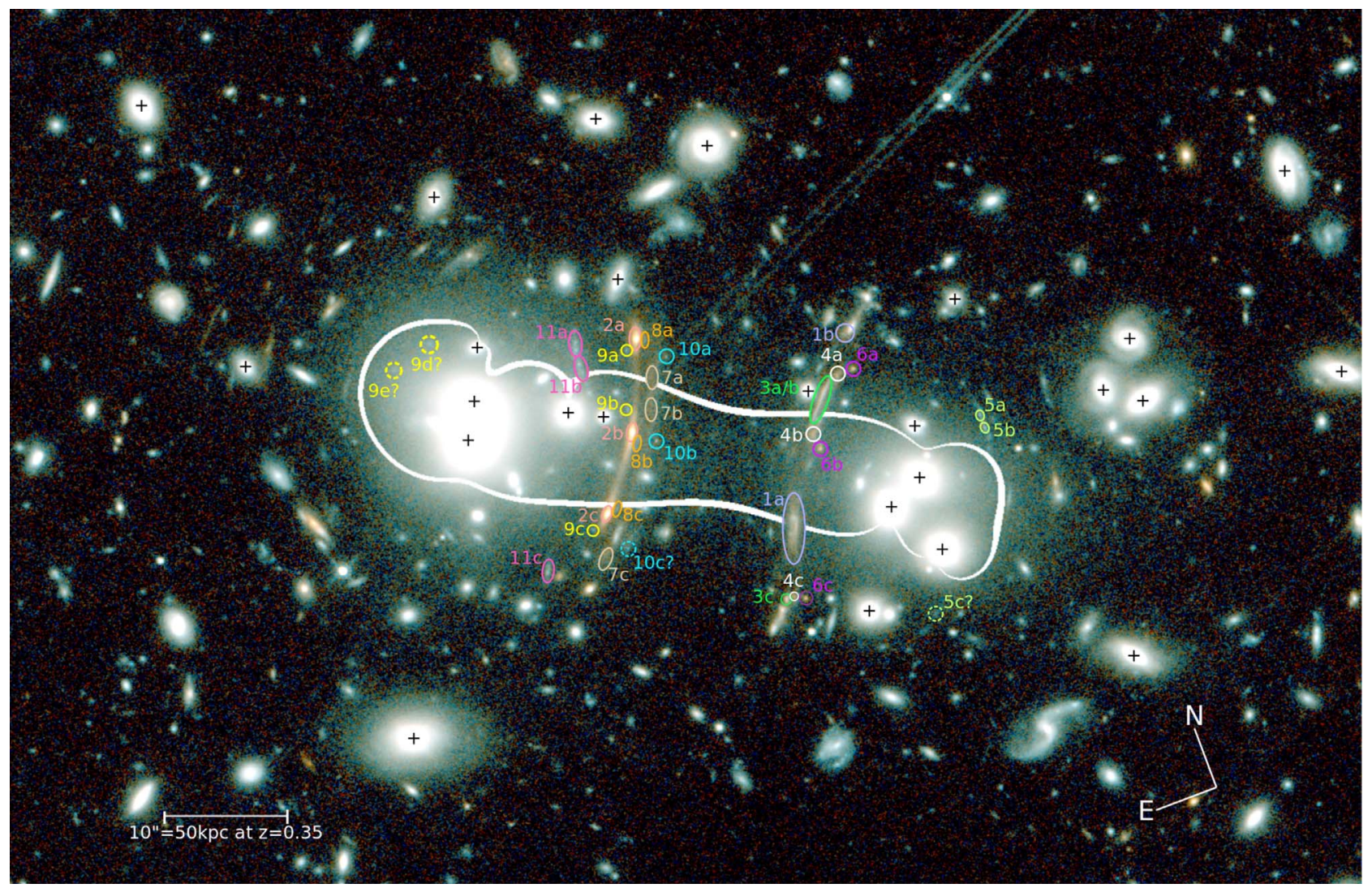

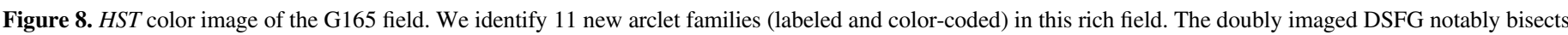

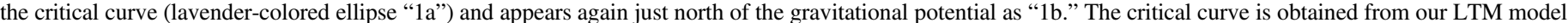

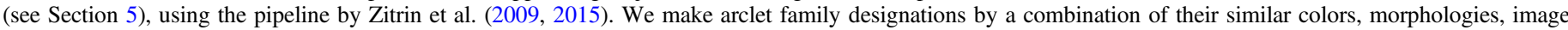
symmetries, and model-predicted locations (see Figure 9 for image stamps).

of $z \simeq 1.5-7$. We expect the redshifts of the arcs roughly to coincide with the star formation rate density peak of the universe of $z=2-3$ (Madau \& Dickinson 2014). Even so, this broader redshift accounts for the potential outliers, as recommended in Johnson \& Sharon (2016) for the case of limited spectroscopic redshift information and also found to be a useful constraint in Cibirka et al. (2018) and Cerny et al. (2018).

The lens model for the G165 cluster field is discussed in detail below, and a lensing analysis for the other five fields appears in Section B.1. We emphasize that all arclet families discovered in this study are supported by our LTM model.

\section{2. $G 165$}

The cluster members form a pattern on the CMD referred to as the red sequence (Gladders \& Yee 2000). The G165 field shows a prominent red sequence characterized by very little scatter, making the designation of the cluster members especially straightforward by applying a color cut in the usual way. At the same time, the amount of scatter increases toward fainter sources owing to the larger photometric errors. To reduce the risk of contamination by objects outside the cluster, we also impose a conservative upper magnitude limit of $\mathrm{F} 160 \mathrm{~W}_{\mathrm{AB}}=21.0$. The initial selection flagged two objects that were found by inspection not to be bona fide cluster members and so were removed. The first was a star, and the second was galaxy "s30" with a spectroscopic redshift of $z=0.135$, which places it in the foreground of the cluster. Our final catalog for G165 uses 38 cluster members in the lens model. The cluster members within the central region found by our blind selection algorithm appear as plus signs in Figure 8. We refer to Appendix B for additional details regarding the cluster member selection.

On the lensing constraints, our HST image is replete with giant arcs and arclet families. The presence of giant arcs, as well as structures consisting of several giant arcs, has been noted before (Cañameras et al. 2015). A preliminary mass model for G165 was made using ground-based CFHT data available at the time (Cañameras et al. 2015, 2018). In total, we present here 11 designations of arclet families, all of which are new to the literature (Table 3). The reference center for our lensing analysis is set to the location of the lensed DSFG at (R.A., decl. $)=(11: 27: 14.731,+42: 28: 22.56)$. By using the positions and brightnesses of the cluster members as constraints in our LTM algorithm, we construct a mass map that is subject to the arclet family constraints. Our model uncovers the two mass peaks evident in the imaging and reproduces all lensed galaxy images with respect to their locations ( $\mathrm{rms} \sim 0$." 65 ). The arclet families are marked on a color image along with the critical curve in Figure 8. Postage stamp images of the arclet family members appear in Figure 9 organized by family name. Below we give a description of each of the 11 arclet family systems, whose properties are also summarized in Table 3.

G165_DSFG_1 (Arcs 1a, 1b)._-Arc 1a is the NIR counterpart of the lensed DSFG at $z=2.2$ detected in the submillimeter data set (Cañameras et al. 2015). This giant arc, which orthogonally bridges the critical curve, has an NIR angular extent of $\sim 5$ !' Our model estimates that Arc $1 a$ is a 


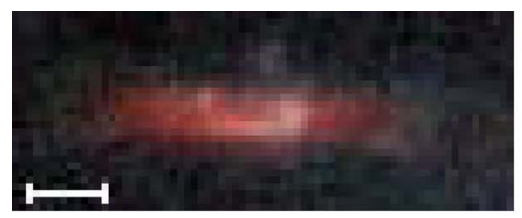

Arc

$1 \mathrm{a}$

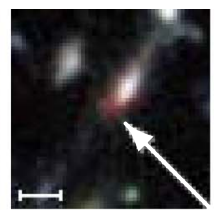

Arc

$1 \mathrm{~b}$

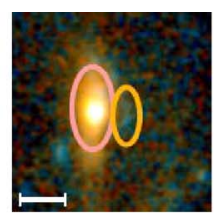

Arc

$2 \mathrm{a}, 8 \mathrm{a}$

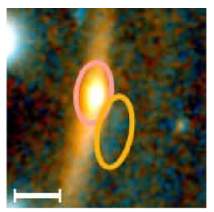

Arcs

$2 \mathrm{~b}, 8 \mathrm{~b}$

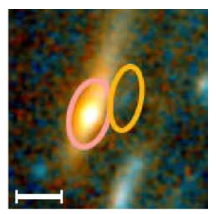

Arcs

$2 \mathrm{c}, 8 \mathrm{c}$

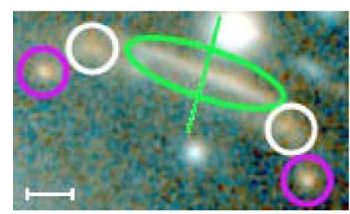

Arcs

$4 \mathrm{~b}, 6 \mathrm{~b}, 3 \mathrm{~b}, 3 \mathrm{a}, 4 \mathrm{a}, 6 \mathrm{a}$

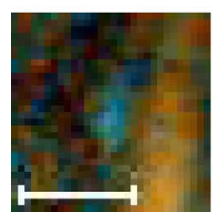

Arcs

$9 \mathrm{a}$

Arcs

$9 \mathrm{~b}$

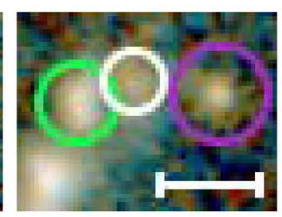

Arcs

$3 \mathrm{c}, 4 \mathrm{c}, 6 \mathrm{c}$

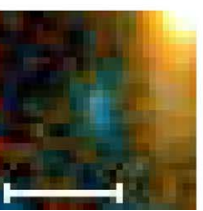

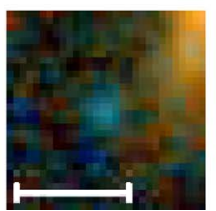

Arcs

9c

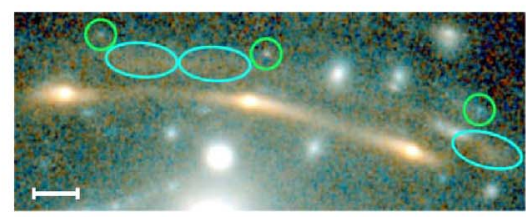

Arcs

10a,7a,7b,10b,10c?,7c

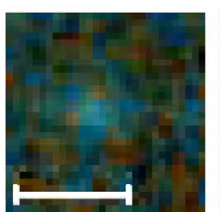

Arcs

$9 \mathrm{~d}$ ?

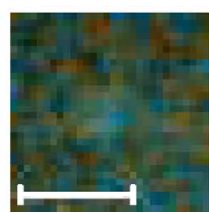

Arcs

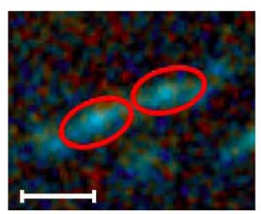

Arcs

$5 \mathrm{a}, 5 \mathrm{~b}$

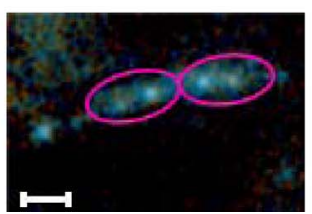

Arcs

$11 \mathrm{a}, 11 \mathrm{~b}$

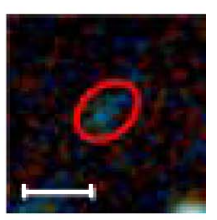

Arcs

$5 c$ ?

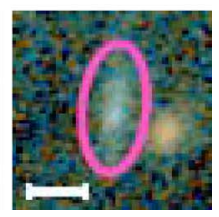

Arcs

$11 \mathrm{c}$

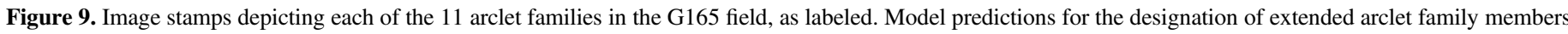

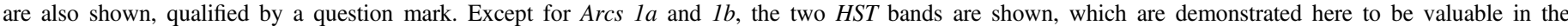

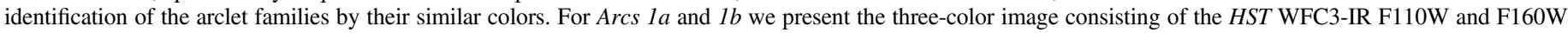

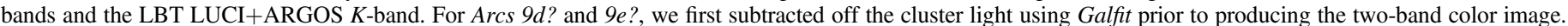

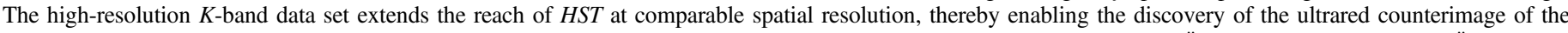

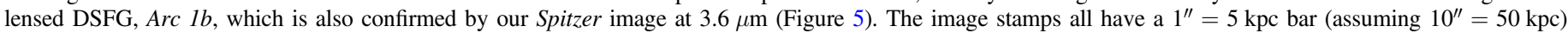
provided for reference. The images maintain the same orientation as in Figure 2.

merging image with a high areal magnification factor of $\gtrsim 30$ that varies along the long axis of the arc. The large areal extent on the sky yields the potential to study properties within its interstellar medium, on physical scales of $\lesssim 1 \mathrm{kpc}$. In particular, two compact and bluer sources appear superimposed onto Arc $1 a$, which are situated on opposite sides of the critical curve at the redshift of the DSFG. These may potentially be images of star-forming knots within the DSFG that are multiply imaged, thereby yielding still higher magnification factors (see Figure 5). Spectroscopy is required to determine the relation of these two blue images to Arc $1 a$. Arc $1 a$ is bright, $K_{A B}=18.74_{-0.02}^{+0.02} \mathrm{mag}$, and well suited for spatially resolved spectroscopic follow-up observations.

A counterimage is also predicted, which is not detected in our HST data set but is detected in the LBT/LUCI + ARGOS $K$-band image at the model-predicted location. A bright image at the exact model-predicted location is also detected in our Spitzer/IRAC imaging data. We designate this arc as the counterimage G165_DSFG_lb (see Figure 5). Interestingly, while the $\mathrm{F} 160 \mathrm{~W}_{\mathrm{AB}}-K_{\mathrm{AB}}$ color is consistent between the two images, there is an offset in the $\mathrm{F} 160 \mathrm{~W}_{\mathrm{AB}}-S_{3.6, A B}$ color by a large $\gtrsim 2.6 \mathrm{mag}$. This color difference is due at least in part to contamination. G165_DSFG_lb appears to be situated behind a bluer and lower-redshift galaxy, which influences the photometry and therefore renders the color unreliable (see Figure 5). G165_DSFG_la is also a merging pair. As such, the background source crosses a cluster caustic such that G165_DSFG_la represents only a region of that background source and only a portion of the starlight. At the same time, $G 165 \_D S F G \_1 b$ unveils the entire source and thus the total integrated galaxy light. It is noteworthy that $G 165 \_D S F G \_1$ is the only arclet family in this field to have a measured spectroscopic redshift. This family is used for the internal minimization or "anchor" of our model.

G165_2a, 2b, 2c (Arcs 2a, 2b, 2c) and G165_8a, 8b, 8c (Arcs $8 a, 8 b, 8 c$ ). - The Arc 2 family members are the brightest in the field, with $K_{\mathrm{AB}}$ magnitudes for each of the three arcs of $\approx 18.5$ mag, making them also excellent sources for follow-up spectroscopy to measure the redshift. For this object, we leave the redshift to be optimized in the modeling. The bluer arclet trio that makes up Arcs 8, which are situated near in projection, is undetected at $K$. Arcs $2 a$ and $2 b$ and Arcs $8 a$ and $8 b$ fold about an axis of symmetry, as do Arcs $7 a$ and $7 b$ and Arcs $10 a$ and $10 \mathrm{~b}$ discussed below.

G165_3a, 3b, 3c (Arcs 3a, 3b, 3c); G165_4a, 4b, 4c (Arcs $4 a, 4 b, 4 c)$; and $6165 \_6 a, 6 b, 6 c(\operatorname{Arcs} 6 a, 6 b, 6 c)$.-For the following description we refer to the close-up image in Figure 5. The family members Arcs $6 a$ and $6 b$ are red and compact arcs that are situated on opposite sides of an axis of symmetry, as marked. Adjacent in projection on the sky, the slightly redder family members Arcs $4 a$ and $4 b$ present more extended morphologies. Coincident with Arcs $4 a$ and $4 b$, the bright family members Arcs $3 a$ and $3 b$ describe a fold arc conjoined at the axis point. The third image of each of these families, Arcs $3 c, 4 c$, and $6 c$, appears at an an angular separation of $\approx 14^{\prime \prime}$. This set of third images for each family retains similar colors and image morphologies and relative image placements.

G165_5a, 5b (Arcs 5a, 5b)._-These faint and blue galaxy images are situated just inside the critical curve and are the only 
secure arclet family members to reside on the opposite side of the gravitational potential. Arcs $5 a$ and $5 b$ are two merging images folded about the critical curve. Meanwhile, the dashed circle labeled as " $5 \mathrm{c}$ ?" marks the position of a candidate counterimage that awaits confirmation pending additional model constraints.

G165_7a, 7b, 7c (Arcs 7a, 7b, 7c); G165_10a,10b (Arcs $10 a, 10 b)$.- Arcs $7 a$ and $7 b$ and Arcs $10 a$ and $10 b$ project onto an arc-like structure that is parallel to Arcs $2 a, 2 b$, and $2 c$. Arcs $7 a$ and $7 b$ are especially red and low in surface brightness. The counterimage that we designate as $A r c 7 c$ appears southward at the model-predicted location. The candidate counterimage labeled as "10c?" appears near to its expected location but at a different color, and so it is not included in our lens model.

G165_9a, 9b, 9c (Arcs 9a, 9b, 9c). - This arclet family trio is distinctively blue and compact. Arcs $9 a$ and $9 b$ are split by an axis of symmetry. Arc $9 c$ appears at the model-predicted location at an angular separation of 10!. Note that two other candidate counterimages are marked in Figure 8 as "9d?" and "9e?" on the opposite side of the gravitational potential, which await confirmation as additional model constraints become available. Although situated near in projection to bright central elliptical galaxies, Arcs 9d? and 9e? are clearly identified in our galaxy-subtracted image using Galfit (Peng et al. 2010) in Figure 9.

G165_11a, 11b, 11c (Arcs 11a, 11b, 11c).-The blue Arcs $11 a$ and $11 b$ are images that merge across the critical curve as indicated by the pair of star-forming knots within Arc 11a that appears again in $A r c 11 b$ with reverse parity. Arc 11c appears at the model-predicted location southeast of the other two arclet family images at an angular separation of $18^{\prime \prime}$.

From our lens model we compute a large effective Einstein radius of $13^{\prime \prime}$ at $z=2.2$ and $16^{\prime \prime}$ at $z=9$. By integrating up the mass surface density, we measure a lensing mass of $(2.6 \pm 0.11) \times 10^{14} M_{\odot}$ within a $\sim 250 \mathrm{kpc}$ radius. By summing up the total area on the magnification map binned by the magnification factor, we compute $A(>\mu)$ as a function of $\mu$. Our profile of this cumulative areal magnification is similar to that of the Weak and Strong Lensing Analysis Package (WSLAP) model of Diego et al. (2007), to within $~ 30 \%$. Note that given the significant visibility of both $G 165 \_D S F G \_l a$ and G165_DSFG_1b in the $K$ band and Spitzer/IRAC, the James Webb Space Telescope (JWST) resolution and sensitivity will be needed at $1-4 \mu \mathrm{m}$ to significantly refine these lens models. We refer to Section 6 for independent measurements of the mass and estimates of the lensing strength and also to Appendix B for the magnification map.

\section{Discussion}

\subsection{The Mass of 6165}

The difference in our values between the lensing and the dynamical masses merits further investigation. Here we discuss our three independent estimates of the mass.

\subsubsection{Lensing Mass}

We measure a lensing mass of $(2.6 \pm 0.11) \times 10^{14} M_{\odot}$ within $\approx 250 \mathrm{kpc}$ by applying the constraints imposed by the 11 arclet families (Section 4.2). Of these, we have spectroscopic confirmation only for G165_DSFG_la of $z=2.2357$ (Harrington et al. 2016). We choose to allow the redshifts of other arclet families to vary as free parameters with values of $z=1.5-7$. While the approach works reasonably well in that it yields accurate model predictions of the counterimages, nevertheless, the lack of redshifts is nonideal. This is because uncertainties in the lensed galaxy redshifts translate into uncertainties on the normalization of the lens model, which in turn lead to changes in the value for the total mass of dark plus visible matter.

We find the mass density to fall off rapidly beyond $250 \mathrm{kpc}$ and to reach $\approx 4 \times 10^{14} M_{\odot}$ within $1 \mathrm{Mpc}$. This value is lower than our value for the dynamical mass by an order of magnitude. This then raises the question whether an external shear component may be situated in such a way that it controls, or at least contributes to, the determination of the positions and orientations of the lensed images. If so, then such a structure could potentially hide additional mass outside of the $H S T$ field of view that would not be accounted for in our strong-lensing mass estimate. There are extended structures in our wider-field $\left(4^{\prime} \times 4^{\prime}\right)$ LBT LUCI-ARGOS $K$-band image, yet our LTM model does not uncover any significant external shear component. At the same time, our model covers only the inner portion of a large and extended lens. Additional deep and wider-field imaging is needed to extend the model into the weak-lensing regime to investigate the influence of any external lensing structures.

\subsubsection{Dynamical Mass}

Our value for the dynamical mass of $1.3_{-0.70}^{+0.04} \times 10^{15} M_{\odot}$ is a factor of $\sim 5$ higher than that of the lensing mass within $250 \mathrm{kpc}$. By making use of our entire spectroscopic data set, which extends to $1 \mathrm{Mpc}$, our value for the dynamical mass remains high, $M_{V}=(9.1 \pm 0.4) \times 10^{15} M_{\odot}$. Relevant to this discussion, the imaging uncovers an obvious bimodal mass structure (Figure 8). If the mass is elongated along the line of sight, then the velocities will also be higher in this direction. In this case the erroneous assumption that the line-of-sight velocity is spherically symmetric will lead to an overestimate of the virial mass. Bimodal masses are not uncommon in massive lensing clusters (e.g., Cerny et al. 2018; Cibirka et al. 2018; Mahler et al. 2018). For example, in Mahler et al. (2018), the two mass peaks appearing in the image of the cluster A2744 are identified spectroscopically in the redshift histogram of 156 cluster members as two velocity peaks separated by $5000 \mathrm{~km} \mathrm{~s}^{-1}$ (their Figure 9). We cannot perform this exercise in our current sample, given the lower numbers of spectroscopic redshifts by an order of magnitude. Instead, we undertake a search for any velocity gradient across the cluster.

We introduce a bifurcation line drawn normal to the line connecting the NE and SW mass peaks at its midpoint (see Figures 8 and 10). We then compute the radial velocities on either side of this line to search for evidence of two velocity peaks to match the incidence of the two mass peaks (Figure 10). But do the velocities give a fair representation of the kinematical structure of the cluster? In a recent paper, Hayashi et al. (2017) report that new cluster members undergoing infall show high line-of-sight velocities at all radii. This is potentially insightful for the G165 field, in which two cluster galaxies have high measured velocities of $v_{\text {los }} \approx 950$ and $1750 \mathrm{~km} \mathrm{~s}^{-1}$ (green open squares in Figure 10). These are also two of only three galaxies showing nebular emission line features indicative of recent star formation. These galaxies have a potentially larger peculiar velocity component and elevated star-forming activities, two attributes that are consistent with 

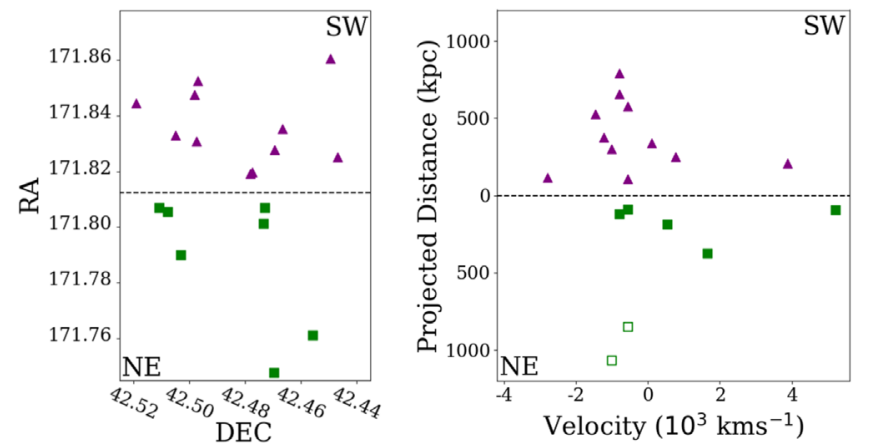

Figure 10. Left: scatter plot of (R.A., decl.) for the cluster members in G165 with spectroscopic redshifts. We divide the cluster into an NE and an SW region by a bifurcation line (dashed) drawn at the midpoint between the two mass peaks. The cluster members on either side of the line are indicated by the square- and triangular-shaped symbols, as marked. Right: scatter plot of the projected distance from the bifurcation line (dashed) as a function of the velocity relative to the cluster mean redshift of $z=0.351$. The two green open squares pertain to objects with high negative velocities and that also show relatively rare nebular emission lines in their spectra. If we remove these two outliers under the assumption that they may represent new infalling galaxies, then the remaining data may suggest a velocity gradient across the cluster. However, using this data set, we cannot place firm constraints on the lensing configuration of G165.

the picture that these objects are infalling members. On consideration of all but these two outliers, there is a hint of a redshift of the NE mass peak relative to the SW one. However, given the small mean velocity difference between the two peaks of $\lesssim 2000 \mathrm{~km} \mathrm{~s}^{-1}$ and the dearth of spectroscopic redshifts, we are unable to constrain the cluster velocity configuration with the current data set. Additional spectroscopy is needed to fill in the sparse redshift sampling to obtain a larger, more representative set of cluster members. We refer to Section 6.3 for the discussion of how the cluster configuration relates to the cluster gas pressure.

\subsubsection{Caustic Mass}

In keeping with the limits typically imposed for the caustic mass estimates, we set a velocity cut of $\pm 5000 \mathrm{~km} \mathrm{~s}^{-1}$ from the mean cluster redshift of $z=0.351$. The redshift information for the 17 of 18 cluster members meeting this requirement provides the input to measure the caustic mass in a formalism developed in Diaferio \& Geller (1997) and Diaferio (1999) (see also Serra et al. 2011; Alpaslan et al. 2012; Windhorst et al. 2018). The approach is to estimate the mass of a cluster of galaxies out to the virial radius by analyzing the distribution of its constituent galaxies in redshift space (i.e., projected separation from the cluster center $R$ as a function of line-of-sight velocity with respect to the cluster median redshift $v_{\text {los }}$ ). On the assumption of a virialized cluster, this distribution resembles the bell of a trumpet (with the spread in $v_{\text {los }}$ increasing at low $R$ ), whose area can be related to the gravitational potential (and hence mass) of the cluster.

It is useful to work in phase space by depicting $v_{\text {los }}$ as a function of their projected distances from the cluster center. We adopt the virialized region from the prescription in Jaffé et al. (2015), such that $v_{\text {los }} \leqslant 1.5 \sigma$ is within a projected distance of $R_{200}$, where $\sigma$ is the velocity dispersion. Indeed, the vast majority of cluster members (black circles in Figure 11) fit well within this radius as depicted by the gray shaded region. We convert our redshift catalog of cluster members into a continuous density field by using an adaptive density kernel.

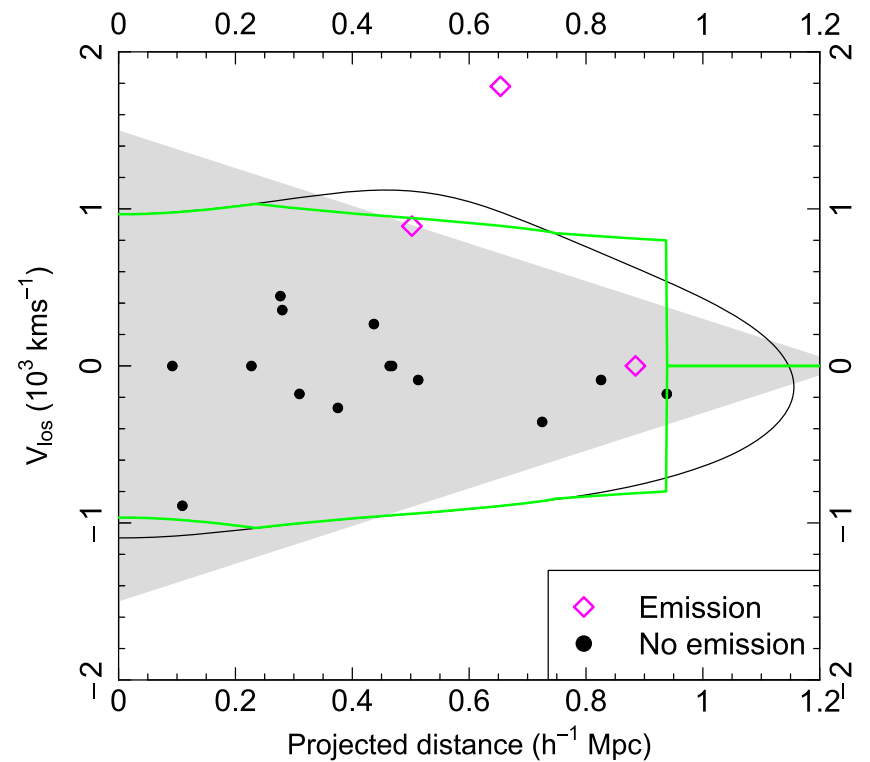

Figure 11. "Trumpet" diagram depicting the caustic mass estimator for G165. We start with the redshift catalog of cluster members (black circles), which we convert into a density field. The contour (black) identifies the region in redshift space at which the galaxy density equates to some threshold value, which in this case corresponds to the escape velocity of the cluster. We rewrite the equation for this threshold density into a form that is symmetric about $v_{\text {los }}=0$ (green "tuning fork") to satisfy the requirement of spherical symmetry in our model. The mass estimate is then made by integrating the area under the caustic lines. We measure a mass of $M_{\text {caustic }}=(1.9 \pm 0.18) \times 10^{15} M_{\odot}$ within $r \approx 0.8 \mathrm{Mpc}$.

The contour (black curve) identifies the region in the redshiftspace distribution that corresponds to the escape velocity of the cluster (assuming spherical infall), which in turn is related to its gravitational potential as $v_{\mathrm{esc}}^{2}=-2 \phi(r)$. In practice, we impose the condition of spherical symmetry by rewriting this density threshold into a symmetric version about the $v_{\text {los }}=0$ line. To do this, we check the absolute values of $v_{\text {los }}$ for this doublevalued function in small increments of radius along the density threshold contour. The caustic equates to the minimum of those two absolute values and is reflected along the $v_{\text {los }}=0$ line to construct the "tuning fork" shape (green contour). The amplitude of the caustic $\mathcal{A}(r)$ is then related to the cluster mass $M$ such that $G M \propto \int_{0}^{r} \mathcal{A}^{2}(r) d r$.

By applying this estimator, we measure a mass of $\approx(1.9 \pm$ $0.18) \times 10^{15} M_{\odot}$ within $0.8 \mathrm{Mpc}$. The uncertainty on this value is derived by a "jackknife" resampling approach consisting of making 20 realizations in which two galaxies at a time are removed at random and the mass recomputed. Analysis of this set yields the stated estimate in the uncertainty of the mass. Note that the mass has been rescaled to be median biased with respect to the dynamical mass, which is calibrated as a function of redshift and cluster richness of comparable systems in Alpaslan et al. (2012). This mass value is a factor of $\sim 5$ higher than the value for the lensing mass extrapolated out to $1 \mathrm{Mpc}$ and a factor of $\sim 5$ lower than the value for the dynamical mass computed within $1 \mathrm{Mpc}$. If G165 does have an aspherical mass distribution elongated along the line of sight (see Section 6.1), then this value will be an overestimate.

\subsection{G165 as a Lens}

We compare the lensing strength of G165 with that of another massive lensing cluster at a similar lens redshift, the 

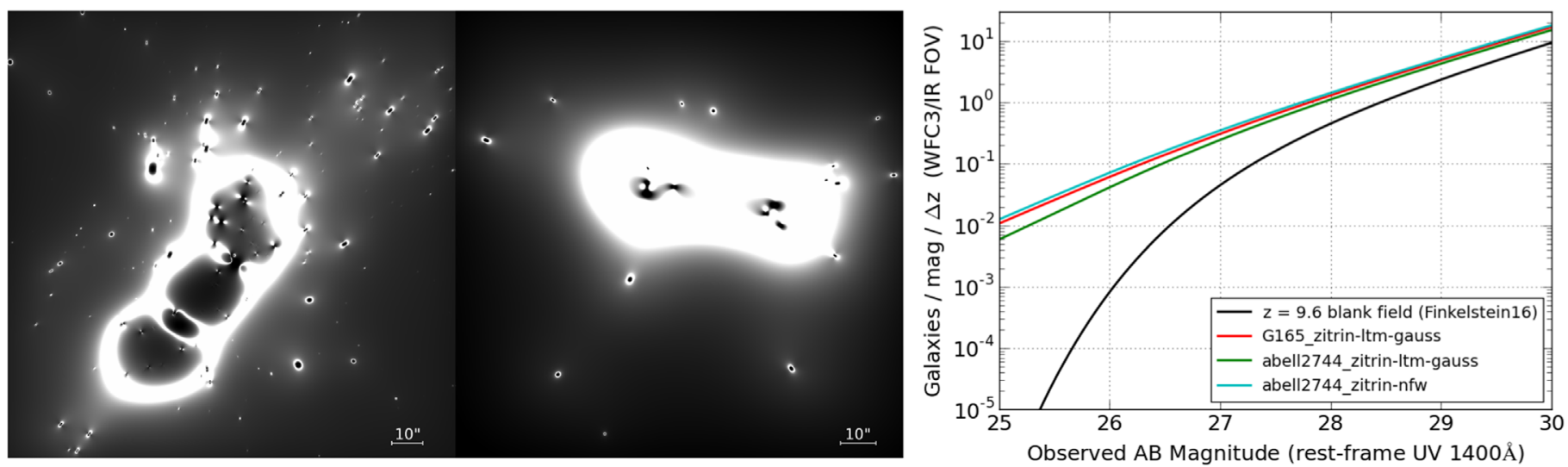

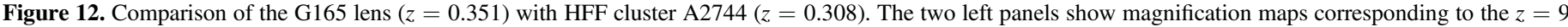

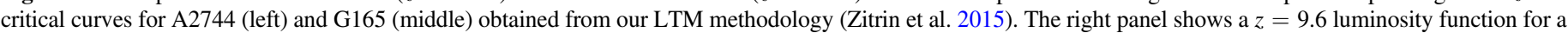

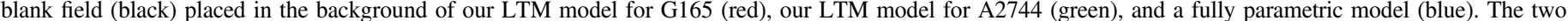

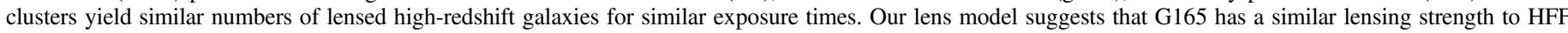

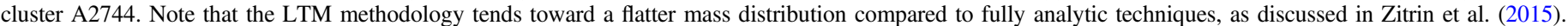

Hubble Frontier Fields cluster A2744 (HFFs; PI: J. Lotz, GO-13495). A2744 provides a useful benchmark for its well-constrained lens model and similar size of its effective Einstein radius. Its strong-lensing model is well constrained with 29 arclet families identified from deep HST imaging in seven bands with $5 \sigma$ limits in each filter of $m_{\mathrm{AB}} \sim 29 \mathrm{mag}$ (Mahler et al. 2018). These limits are $\sim 1.3$ and $\sim 1.8 \mathrm{AB}$ mag deeper than the $5 \sigma$ limiting magnitudes for G165 of $\mathrm{F} 110 \mathrm{~W}_{\mathrm{AB}}=27.7 \mathrm{mag}$ and $\mathrm{F} 160 \mathrm{~W}_{\mathrm{AB}}=27.0 \mathrm{mag}$. For consistency, we construct the models for both clusters by our LTM approach, where the lens model for G165 comes from this paper and the one for A2744 is from Zitrin et al. (2014). We show the lens models in the two leftmost panels in Figure 12. In both cases we find a similar elongated shape and effective $z=9$ critical curve size of $\sim 16^{\prime \prime}$. To compute the lensing strengths, we assume the same background luminosity function (Finkelstein 2016) and then compare the number distribution of lensed background galaxies in the two fields. Overall, the clusters G165 and A2744 yield significantly brighter objects compared to a blank field at all magnitudes. At high redshifts, the clusters G165 and A2744 yield on average similar numbers of $z \approx 9.6$ objects (right panel in Figure 12).

G165 is an ideal lens through which to investigate highredshift objects $(z=9-12)$. This is in part due to the relatively low redshift of the lens plane of $z=0.351$, for which the level of the intracluster light (ICL) contamination at the NIR wavelengths corresponding to the Lyman break for $z>9$ galaxies is minimized (Windhorst et al. 2018). G165 also has a reasonably high ecliptic latitude of $35^{\circ}$, reducing its background from the peak with the zodiacal plane. The lens size is ideal for JWST/NIRCam imaging, as the lens fills (but does not overfill) the field of view out to $\sim 3$ times its Einstein radius. Note that for relatively shallow exposures typical of a JWST short program reaching limiting fluxes of $m_{\mathrm{AB}} \sim 28 \mathrm{mag}$, the improvement of JWST will be seen at all wavelengths. The imaging at $\lambda>1.6 \mu \mathrm{m}$ will enable robust detections of the stellar continuum of any new high-redshift galaxy candidates situated behind lensing clusters.

We consider the potential for G165_DSFG_la to yield caustic transit events. In its favor, two compact and bluer knots appear in projection to be within this arc that bridges the critical curve and have small angular separations from the critical curve of roughly tenths of an arcsecond (see Figures 5 and 9).
Unlike distributed masses that incur magnification factors of up to $\mu=40-50$, compact sources such as stars and star clusters can reach magnifications of $\mu \lesssim 10^{5}$ (Windhorst et al. 2018). To assess the practicality of monitoring this arc for caustic transits, we require in addition a negligible ICL component. Diego et al. (2018) showed how, if the ICL at the position of the critical curve is significant, the stars from the ICL can set an upper limit (through microlensing) on the maximum magnification of background stars during caustic crossing events to around $10^{4}$. Since the ICL can extend up to large distances from the center of the halos (see, e.g., Mihos 2016), even critical curves that are relatively far from the center of halos could be affected by the microlensing from the ICL stars.

\subsection{The G165 Cluster Gas Pressure}

Given the somewhat novel search strategy to find the G165, it is natural to ask how this massive lensing cluster compares with others selected by more commonly used methods, such as X-ray brightness or the detection of the SZ decrement. G165 has high mass and high dark matter concentration, as evidenced by the prominent displays of giant arcs and arclet families even in these relatively shallow (single-orbit) HST images. As such, we would expect G165 to be replete with large amounts of cluster gas.

G165 is in fact undetected in ROSAT imaging (R6+R7 bands, or $\sim 0.7-2 \mathrm{keV})$. Put another way, G165 is at best a lowluminosity X-ray source with an upper limit on its X-ray flux computed from the RASS diffuse map of $1.12 \times 10^{-4}$ counts $\mathrm{s}^{-1} \operatorname{arcmin}^{-2}$. It is unusual for a truly relaxed cluster to have an $\mathrm{X}$-ray flux so low as to be undetected by ROSAT at this redshift and mass scale. At the same time, at these lower luminosities, the scaling relations correlating the X-ray luminosity to cluster mass are more uncertain owing to a large intrinsic scatter in the data (Bruch et al. 2010). G165 also misses out on membership in the Planck Sunyaev-Zel'dovich (PSZ) cluster catalog as a result of its low SZ signal, which falls below the minimum detection threshold. In the Planck Compton- $Y$ parameter map there is a small fluctuation at the position of the cluster that may represent a weak detection of intercluster gas, or it may be noise given that the detection is only at the $1 \sigma-2 \sigma$ level. This lack of a significant SZ signal might be a consequence of radio 
emission washing out a shallow decrement, projection effects, or an overestimation of the cluster mass.

Radio sources have an inverted spectrum with respect to IR sources that can counteract the SZ signal. AsDSFG_G165_la is the one image in the field with high submillimeter flux arising from high star formation and/or AGN activity; this lensed DSFG is the most likely source to be radio-loud. There is a weak radio emitter detected near the position of the IR source. From NVSS data we measure a total flux from the cluster including this IR source of $<40 \mathrm{mJy}$ at $1.4 \mathrm{GHz}$ (Condon et al. 1995). Although present, this modest radio signal is insufficient to compete with the SZ effect at the relevant frequencies $(100-353 \mathrm{GHz})$, thereby ruling out radio contamination as an explanation for the relative SZ silence.

The last conventional explanation is the lensing configuration. The G165 field contains an obvious bimodal substructure. There are other examples of bimodal mass structures, such as the well-studied Bullet Cluster (Bradač et al. 2006; Clowe et al. 2006) and the cluster known as "El Gordo" (Menanteau et al. 2012). These two clusters are classified as "post-mergers" that, in turn, produce significant enhancements of the X-ray flux. If the field is elongated along the line-of-sight direction as a series of two smaller galaxy structures, then we may be catching G165 during a less well studied evolutionary "pre-merger" phase. In this scenario, the total cluster gas pressure dilutes across the large structure, which reduces the gas pressure and the X-ray emission, hence reducing the SZ decrement. At the same time, the mass integrated along the line of sight still provides ample surface mass densities, leading to stronglensing effects. A test of this hypothesis can be made by acquiring $\mathrm{X}$-ray observations. For example, $X M M$ imaging at the level of 20-27 ks total exposure allows us to measure the distribution and centroid and place limits on the electron temperature of the X-ray gas. If G165 deviates from a monolithic mass, then an offset will be detected, or at least an X-ray source that is marginally extended yet still potentially offset from the center of mass.

\section{Summary}

Searching wide-field imaging data sets for giant arcs is now fairly common, yet conducting searches for unresolved giant arcs at submillimeter wavelengths is still relatively rare. We obtained HST WFC3-IR imaging of the fields of six lensed DSFGs selected in a novel search by their rest-frame FIR color and compactness using Planck/Herschel data. We extend the analysis on the G165 field, which shows prominent giant arcs and arclet families. We find the following:

1. Each of our six sample fields unveils the HST WFC3-IR counterpart of the strongly lensed DSFG. In four fields, the DSFG image appears as an image multiplicity at $H S T$ resolution (G165, G045, G145, and G080).

2. For the G165 field, we obtained ground-based spectroscopy using MMT/Hectospec and Gemini/GMOS. We measure 51 new redshifts, which augment the spectroscopic catalog of objects in this field by a factor of five. For the five cluster members within $250 \mathrm{kpc}$, we compute a velocity dispersion and then apply the virial theorem to estimate the dynamical mass of $1.3_{-0.70}^{+0.04} \times 10^{15} M_{\odot}$. Using our full catalog of 18 cluster members, we compute a dynamical mass of $M_{V}=(9.1 \pm 0.4) \times 10^{15} M_{\odot}$ within $1 \mathrm{Mpc}$. We also estimate the caustic mass, which is
$(1.9 \pm 0.18) \times 10^{15} M_{\odot}$ within $\sim 0.8 \mathrm{Mpc}$. These mass estimates are high, possibly owing to enhanced velocity structure in the line-of-sight direction and/or several nonvirialized cluster substructures adding to the lensing power.

3. For the G165 field, we acquired LBT LUCI+ARGOS $K$-band imaging at high resolution $(\mathrm{FWHM} \approx 0$." 3 ). The $K$-band image uncovers dozens of lensed galaxies, including 11 arcs drawn from five different arclet families. We confirm the image position of the lensed DSFG, Arc 1a. We also make the first detection of its counterpart, Arc $1 b$, at the model-predicted location, which is too faint and too red to detect in our HST data set.

4. In total, for the G165 field we identify 11 arclet families by their similar colors and morphologies, which are all new. Obvious axes of symmetry corroborate our arclet family designations. In the NIR, Arc la subtends $5^{\prime \prime}$ and is magnified by a factor of $\gtrsim 30$. Arc $1 b$ is fainter and detected only in our high-resolution LBT/LUCI + ARGOS $K$-band image and Spitzer/IRAC images. We measure an $\mathrm{F} 160 \mathrm{~W}_{\mathrm{AB}}-S_{3.6}$ color difference between the two images that arises because Arc $1 a$ is a merging image and so represents only a portion of that background source, while Arc $1 b$ uncovers the entire source image.

5. We use the LTM approach to construct a mass map in the fields for which there is at least one arclet family detected in our data set (G165, G045, G145, and G080). For the cases without arclet families, we generate a $\kappa$-map through the galaxy brightnesses and orientations. For G165, we estimate a lensing mass of $(2.6 \pm 0.11) \times 10^{14} M_{\odot}$ within $\sim 250 \mathrm{kpc}$. We compute effective Einstein radii for G165 of $\sim 13^{\prime \prime}$ at $z=2.2$ and $\sim 16^{\prime \prime}$ at $z=9$.

6. The lensing properties for G165 are not far different from those of other well-studied massive lensing clusters. In a counting simulation, for G165 we predict comparable numbers of high-redshift objects to $z \sim 9.6$ to those of A2744, another well-studied lensing cluster with similar lens redshift and dark matter properties.

7. Based on the 18 spectroscopic redshifts of cluster members in G165, we currently lack the number of redshifts to distinguish convincingly bimodality in the velocity distribution. Confirmation of a line-of-sight configuration is impactful because it can help to explain the weak X-ray and SZ effect detections. This is because a line-of-sight orientation will dilute the intercluster gas below the ROSAT and PSZ effect detection limits, while maintaining a high surface mass density integrated over the line of sight that amply suffices to explain the observed strong-lensing effects.

We appreciate helpful discussions with Eiichi Egami, Xiaohui Fan, Dan Marrone, Ann Zabludoff, and Scott Tremaine, and we especially thank the anonymous referee for comments that significantly improved this paper. Support for program HST GO-14223 was provided by NASA through a grant from the Space Telescope Science Institute, which is operated by the Association of Universities for Research in Astronomy, Inc., under NASA contract NAS5-26555. J.M.D. acknowledges the support of project AYA2015-64508-P (MINECO/FEDER, UE). R.A.W. was funded by NASA JWST Interdisciplinary Scientist grants NAG5-12460, NNX14AN10G, and 80GNSSC18K0200 from NASA Goddard Space Flight Center. M.P. and A.B. were 
funded by a UA/NASA Space Grant for Undergraduate Research. This work is based in part on observations made with the Spitzer Space Telescope, which is operated by the Jet Propulsion Laboratory, California Institute of Technology, under a contract with NASA. This work makes use of the Large Binocular Telescope, which is an international collaboration among institutions in the United States, Italy, and Germany. We would like to thank the staff at Gemini-North and at the MMT for performing the observations in service mode.

\section{Appendix A \\ NIR Counterparts of Our Lensed DSFG Sample}

We searched for the NIR counterparts of the DSFG submillimeter sources. Using the submillimeter positions as a guide, we detect red and relatively bright NIR counterparts for all six lensed DSFGs at the expected locations with respect to their positions in the submillimeter data (Cañameras et al. 2015, their Figure 2). In all cases, the lensed DSFG images in the HST images stand out as the reddest sources in the field. Note that these galaxy images are significantly magnified, even if their size is smaller than or equal to the instrumental resolution of $H S T$. Despite their small angular extents in some cases, these lensed sources are still among the brightest DSFGs in the sky in the NIR owing to their large estimated magnification factors.

In the G145, G165, G045, and G080 fields, we detect multiple images of a single background DSFG. For G145 and G080, we find that two of the images match up with peaks in the submillimeter (the plus signs in Figure 2). In another field, G092, the NIR counterparts are also identified but show different morphologies despite their similar colors. These images are more likely to be two unrelated and possibly interacting DSFGs at a similar redshift (see Section B.5). For G244, we detect the submillimeter arc but do not spatially resolve the Einstein ring structure, although two sets of arclet families are identified in this field using high-resolution ALMA data (Cañameras et al. 2017a, 2017b). Finally, for G165 we find that G165_DSFG_la bridges the critical curve. We detect another red source at the model-predicted location of the counterimage, G165_DSFG_1b, that is prominent in our LBT/ LUCI+ARGOS $K$ image and in Spitzer/IRAC (dashed circle in Figure 5). The colors between the two images are different, which was initially unexpected, as lensing is achromatic. At the same time, G165_DSFG_la is an arc that is merging with an image of itself. Here the background source is crossing a cluster caustic, such that G165_DSFG_la represents only a portion of that background source, while G165_DSFG_lb shows the entire source (see Section 5.2 for more details). The estimation of the strong-lensing properties appears below.

\section{Appendix B Lensing Analysis}

We apply our well-tested LTM pipeline to the G045, G145, G092, G080, and G244 fields, and we refer the reader to Section 5.2 for the details concerning the lens model for G165. For each field, the red lensing galaxies populate a distinctive region of the CMD in Figure 13. Galaxies on this "red sequence" have a similar color because they have a similar redshift and share a similar star formation history. This NIR color captures the ellipticals on the slowly varying part of the observed-frame SED of a several-gigayear-old elliptical galaxy, such that by applying a blind color cut to the HST F110W and F160W data, the red sequence is easily established in each field (red filled stars in Figure 13). To reduce the chances for contamination from foreground/background objects, we impose a conservative magnitude cut in the range of $\mathrm{F} 110 \mathrm{~W}_{\mathrm{AB}}=21-22 \mathrm{mag}$, depending on the field. We have spectroscopy within the HST field of view for four clusters, G165, G045, G145, and G080, which aids further in their identification (gold filled circles in Figure 13). Using as inputs the positions and brightnesses of the cluster members and the positions and orientations of the arclet families, we construct the strong-lensing model for each field. We emphasize that all arclet families discovered, which include the lensing fields G165, G145, G045, and G080, are supported by our LTM model. The resulting 2D magnification maps are plotted as ratios of the local surface mass density over the critical surface mass density, or $\kappa$-map, in Figure 14. We refer to Table 2 for a summary of the redshifts of the lenses, the lensed DSFGs, and other relevant information.

\section{B.1. $G 145$}

The positional centroids from the submillimeter image are indicated by the gold plus signs in Figure 2. We find NIR counterparts for two of these three peaks, which we designate as G145_DSFG_la and G145_DSFG_lb. These two small arcs are only marginally resolved using $H S T$. Initially, only one counterpart image was identified, DSFG_G145_la. A careful search unveiled a second image with a similar color, at the modelpredicted location, which we designate as $D S F G \_G 145 \_l b$. Using these two arcs as inputs, the model predicts a third image that coincides with the image in the submillimeter but is not detected by HST. The lack of a detection is not surprising, given the faintness of the other two NIR counterparts, which both hover around the limiting magnitude of our observations. The redshift distribution of galaxies in this field is broad, with a somewhat poorly defined peak at $z \approx 0.837$, which we take to be the lens plane. This value is based on four redshifts in the $3 \sigma$ clipped range $0.822<z<0.852$ drawn from our spectroscopy, which all fall within the HST field of view (gold filled circles in Figure 13). This spectroscopy will appear in a separate paper. We note that there is no spectroscopic information available from data archives or other sources. The redshifts for the four lensing members that are situated within our HST field of view appear as the gold filled circles in Figure 13. Our lens model recovers both the image positions and angular separations of the counterimages with an rms of $\sim 0$ ! 1 . In turn, we estimate magnification factors of $12 \pm 0.5$ and $5 \pm 0.5$ for $G 145 \_D S F G \_l a$ and $G 145 \_D S F G \_1 b$, respectively. We estimate the uncertainty by sampling the values for the magnification in a neighboring annular region of width $2^{\prime \prime}$, an approach that works reasonably well for images that are not very near in projection to the critical curve ( $\gtrsim$ few arcseconds). Our model yields effective Einstein radii of $10^{\prime \prime}$ at the redshift of the lensed DSFG and $9^{\prime \prime}$ at $z=9$.

\section{B.2. $G 244$}

We confirm the NIR counterpart of the lensed DSFG as a red and spatially extended image, although the ring-like structure and two arclet families seen in the ALMA data are blended with the primary lens in our HST image and are thus unresolved (Figure 2). The primary lensing galaxy consists of a single object with a measured redshift of $z=1.5$, which also blended 

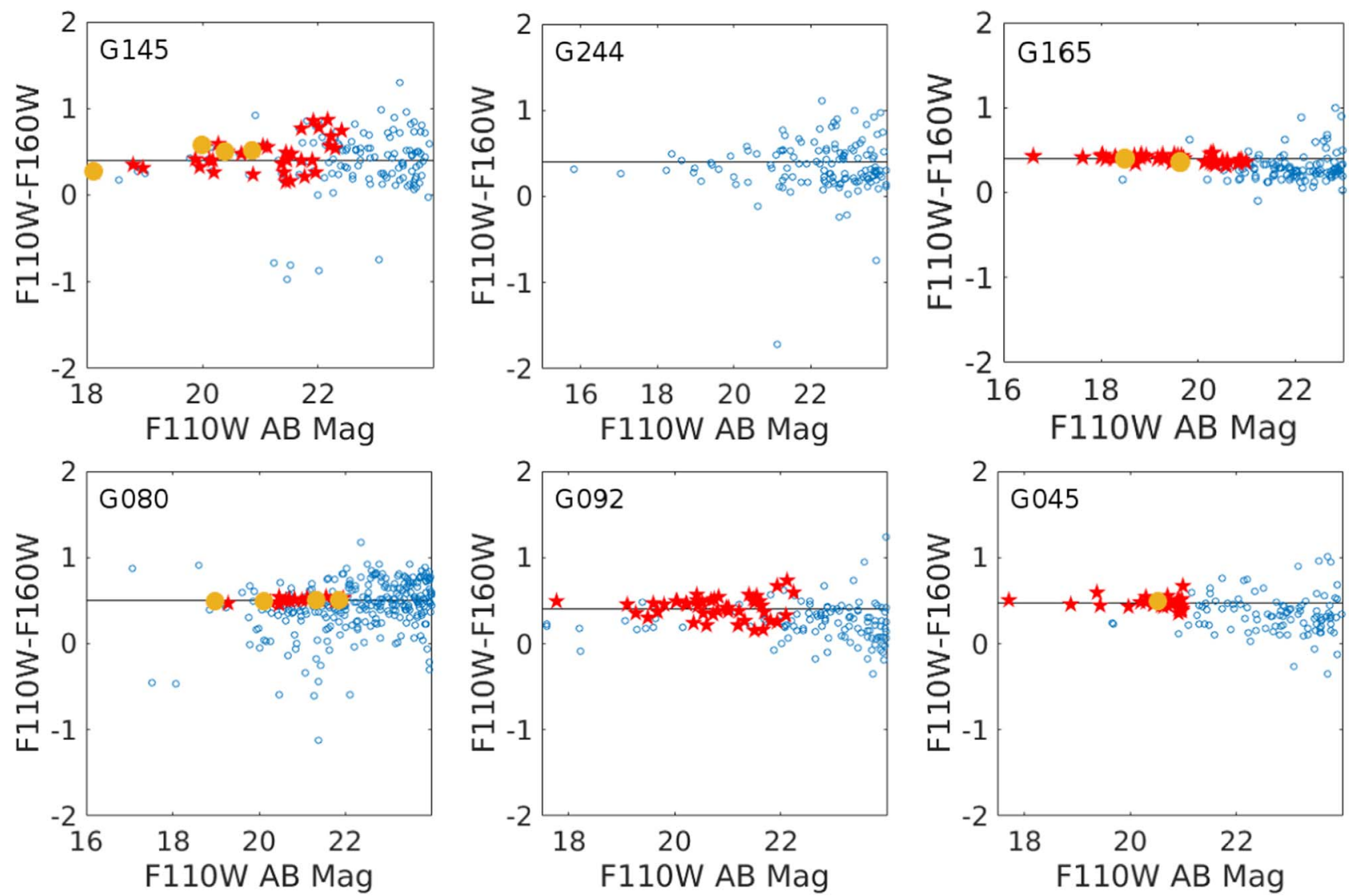

Figure 13. CMDs in the six sample fields computed from our HST/WFC3-IR photometry (blue open circles). The cluster members used in our lens models are indicated by the red filled stars, and the objects with measured spectroscopic redshifts in each cluster that are also situated in our HST images are depicted by the gold filled circles. A fiducial line centered on the red sequence of each lensing field is shown for reference (black solid line). The G165 field shows a tight red sequence despite the obvious bimodal distribution of the mass, possibly indicating that these early-type galaxies share a similar star formation history.

with the lensed DSFG. Elsewhere in the field there are two blue arcs in the near projected proximity of the brightest cluster galaxy that appear to be unrelated images, and no other arclet families are identified. Without arclet families we cannot construct a lens model for this field. Based on this information, we are able to approximate the surface mass density relative to the critical value through the relative galaxy brightnesses and orientations to yield a $\kappa$-map (Figure 14), yet we do not cite a $z=9$ critical curve radius. Note that this field already has a published model based on the ALMA data (Cañameras et al. 2017a, 2017b).

\section{B.3. $G 045$}

Four peaks of the lensed DSFG are detected in the submillimeter and ALMA imaging (Cañameras et al. 2015; Nesvadba et al. 2016). Of these, we find NIR counterparts for three images, which we designate here as $G 045 \_D S F G \_l a, 1 b$, and $1 c$ (see Figure 2). We measure a spectroscopic redshift for the lens of $z=0.556$, based on seven redshifts in the $3 \sigma$ clipped range $0.535<z<0.577$ drawn from our spectroscopy, which will appear in a separate paper. Of these, the redshift for one cluster member is situated within the field of view of our HST data (gold filled circles in Figure 13). Our lens model recovers both the image positions and angular separations of the counterimages with an rms of $\sim 0$ ". 4 . In turn, the model yields high magnification factors of $\gtrsim 9, \gtrsim 9$, and $\gtrsim 7$ for G045_DSFG_la, $1 b$, and $1 c$, respectively. In an independent analysis, the magnification factors of $10-22$ were measured for smaller emission-line regions within each arc (Nesvadba et al. 2016). We compute effective Einstein radii of $8^{\prime \prime}$ at the lensed DSFG redshift and $10^{\prime \prime}$ at $z=9$.

\section{B.4. $G 080$}

The submillimeter imaging shows three bright peaks of this one lensed DSFG. The positional centroids of the peaks are indicated in Figure 2 by the gold plus signs and labels. We designate the two NIR counterparts that we detect in our HST imaging as G080_DSFG_la and G080_DSFG_lb. There is considerable noise at the expected positions of the images owing to their close projected proximity to the extended halos of bright lensing galaxy members. We found that by smoothing the data we were able to take out the high-contrast noise, an exercise that enabled the detection of G080_DSFG_la and G080_DSFG_lb (see inset of Figure 2). Interestingly, we measure a shift by up to $0 . " 5$ in the positional centroids of G080_DSFG_la and G080_DSFG_lb between the SMA and HST images, equating to a physical extent in the source plane of $\sim 4 \mathrm{kpc}$. We find no good explanation for these positional offsets. We measure a lens redshift of $z=0.670$ that is based on 10 redshifts in the $3 \sigma$ clipped range $0.649<z<0.691$ drawn from our spectroscopy in this field, whose results will appear in an upcoming paper. Of these, the redshifts of four of the cluster members are situated within the field of view of our $H S T$ data (gold filled circles in Figure 13). In general, the red sequence shows somewhat more scatter than in some of the other fields, which introduces a higher probability for 

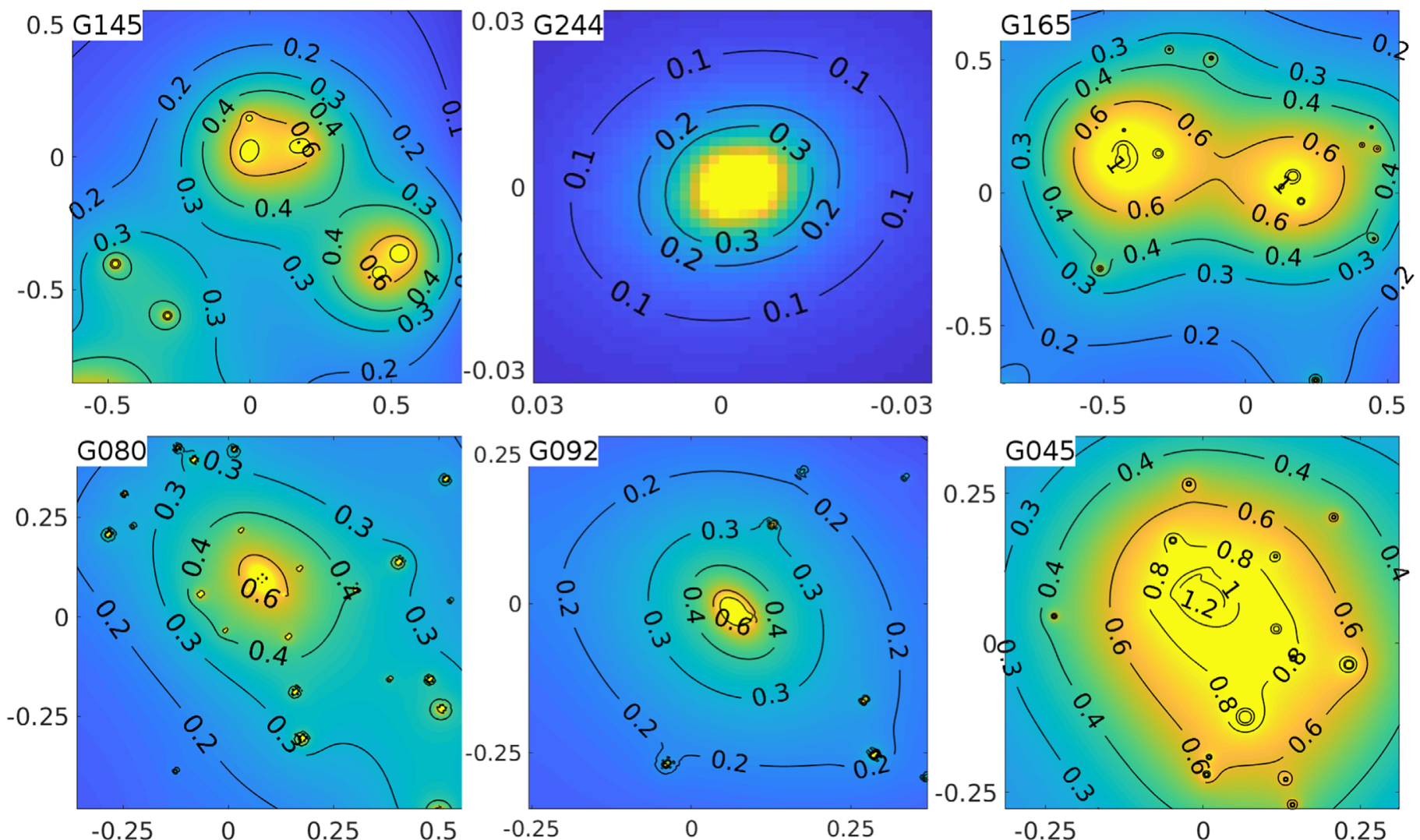

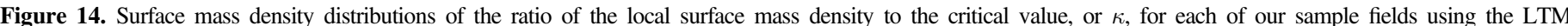

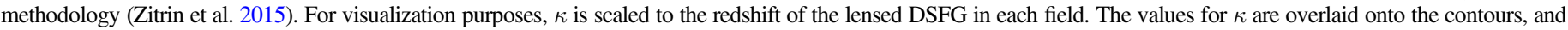

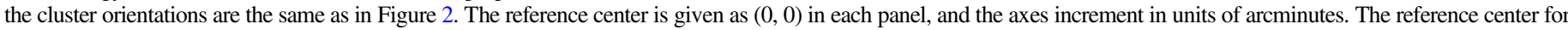

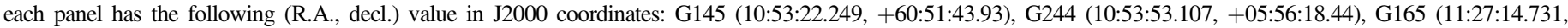
$+42: 28: 22.56)$, G045 (15:02:36.012, +29:20:50.51), G080 (15:44:33.202, +50:23:43.53), and G092 (16:09:17.842, +60:45:19.41).

misidentifying objects of roughly similar colors. To mitigate any potential contamination by galaxies external to the cluster, we make a conservative color cut, resulting in a narrow band of cluster members (red filled stars in Figure 13), yet the uncertainty on the placement of this narrow color cut ultimately limits its usefulness. Our lens model recovers both the image positions and angular separations of the counterimages with an rms of $\sim 2$ !" 2 . The relatively low value of the rms uncertainty shows that the model is robust. At the same time, the rms value is higher than those computed for the other fields in our sample for two reasons: (1) there is a higher uncertainty on the positional centroids of $G 080 \_D S F G \_1 a$ and $G 080 \_D S F G \_1 b$ given their ultralow surface brightness, and (2) the high scatter in the red sequence translates into a higher probability for contamination by objects with similar colors that are not bona fide lensing galaxy members. From our lens model high magnification factors of $\sim 20$ are measured for each of the two images. We compute effective Einstein radii of $\sim 7^{\prime \prime}$ at the redshift of the lensed DSFG and $\sim 11^{\prime \prime}$ at $z=9$.

\section{B.5. $G 092$}

The single "tadpole-shaped" arc detected in the SMA imaging breaks up into two lensed sources, G092_DSFG_la and $G 092 \_D S F G \_1 b$, in our HST images. These arcs are not easily reproduced by our lens model despite their similar colors. A clue to their nature is given by subtracting off the light of the central elliptical galaxy using Galfit. By doing this, we uncover significant differences in the smooth versus clumpy components of the two images (Figure 2, inset). The measured redshift is $z=3.3$, which is integrated over both components. Based on the available information, we infer that these two images are two different galaxies at a similar redshift. As such, this may potentially be an example of a pair of interacting galaxies that induces the ultrahigh star formation rates of $\sim 1000 M_{\odot} \mathrm{yr}^{-1}$ obtained from correcting the value in Cañameras et al. (2015, their Table 2) by the magnification factor provided from our lens model. There is only a single available redshift in this field from the literature, which is of high value, as it corresponds to that of the central lensing galaxy $(z=0.448$ from SDSS DR 14). Without an arclet family, we cannot construct a robust lens model for this field. At the same time, we are able to approximate the surface mass density relative to the critical value through the relative galaxy brightnesses and orientations to yield a $\kappa$-map (Figure 14). By adopting our best-fit scenario that $G 092 \_D S F G \_1 a$ and $G 092 \_D S F G \_1 b$ are two singly imaged lensed sources at a similar redshift, we compute high magnification factors of $\sim 20$ for each image, and we do not cite a $z=9$ critical curve radius.

\section{ORCID iDs}

Brenda L. Frye (iD https://orcid.org/0000-0003-1625-8009

Adi Zitrin (1) https://orcid.org/0000-0002-0350-4488

Greg Walth (10) https://orcid.org/0000-0002-6313-6808 
Haojing Yan (1) https://orcid.org/0000-0001-7592-7714 Christopher J. Conselice (1) https://orcid.org/0000-00031949-7638

Mehmet Alpaslan (ㄷ https://orcid.org/0000-0003-0321-1033

Dan Coe (1) https://orcid.org/0000-0001-7410-7669

Megan Donahue (10 https://orcid.org/0000-0002-2808-0853

Rolf A. Jansen (1) https://orcid.org/0000-0003-1268-5230

Rachael Livermore (1) https://orcid.org/0000-0003-4456-1566

Rogier A. Windhorst (1) https://orcid.org/0000-0001-8156-6281

\section{References}

Acebron, A., Cibirka, N., Zitrin, A., et al. 2018, ApJ, 858, 42

Allen, S. W., Evrard, A. E., \& Mantz, A. B. 2011, ARA\&A, 49, 409

Alpaslan, M., Robotham, A. S. G., Driver, S., et al. 2012, MNRAS, 426, 2832

Bahcall, N. A. 1977, ARA\&A, 15, 505

Benson, B. A., de Haan, T., Dudley, J. P., et al. 2013, ApJ, 763, 147

Bertin, E., \& Arnouts, S. 1996, A\&AS, 117, 393

Blain, A. W. 1999, MNRAS, 309, 955

Bleem, L. E., Stalder, B., de Haan, T., et al. 2015, ApJS, 216, 27

Bradač, M., Clowe, D., Gonzalez, A. H., et al. 2006, ApJ, 652, 937

Broadhurst, T., Benítez, N., Coe, D., et al. 2005, ApJ, 621, 53

Broadhurst, T., Huang, X., Frye, B., \& Ellis, R. 2000, ApJL, 534, L15

Bruch, S., Donahue, M., Voit, G. M., Sun, M., \& Conselice, C. J. 2010, ApJ, 724, 608

Bussmann, R. S., Pérez-Fournon, I., Amber, S., et al. 2013, ApJ, 779, 25

Calanog, J. A., Fu, H., Cooray, A., et al. 2014, ApJ, 797, 138

Cañameras, R., McKenzie, T., König, S., et al. 2015, A\&A, 581, A105

Cañameras, R., Nesvadba, N., Kneissl, R., et al. 2017a, A\&A, 600, L3

Cañameras, R., Nesvadba, N., Kneissl, R., et al. 2017b, A\&A, 604, A117

Cañameras, R., Nesvadba, N., Kneissl, R., et al. 2018, A\&A, 620, A60

Carlstrom, J. E., Ade, P. A. R., Aird, K. A., et al. 2011, PASP, 123, 568

Casey, C. M., Narayanan, D., \& Cooray, A. 2014, PhR, 541, 45

Cerny, C., Sharon, K., Andrade-Santos, F., et al. 2018, ApJ, 859, 159

Cibirka, N., Acebron, A., Zitrin, A., et al. 2018, ApJ, 863, 145

Clowe, D., Bradač, M., Gonzalez, A. H., et al. 2006, ApJL, 648, L109

Condon, J. J., Anderson, E., \& Broderick, J. J. 1995, AJ, 109, 2318

Diaferio, A. 1999, MNRAS, 309, 610

Diaferio, A., \& Geller, M. J. 1997, ApJ, 481, 633

Díaz-Sánchez, A., Iglesias-Groth, S., Rebolo, R., \& Dannerbauer, H. 2017, ApJL, 843, L22

Diego, J. M., Kaiser, N., Broadhurst, T., et al. 2018, ApJ, 857, 25

Diego, J. M., Tegmark, M., Protopapas, P., \& Sandvik, H. B. 2007, MNRAS, 375, 958

Dole, H., Lagache, G., Puget, J.-L., et al. 2006, A\&A, 451, 417

Ebeling, H., Barrett, E., Donovan, D., et al. 2007, ApJL, 661, L33

Ebeling, H., Edge, A. C., Mantz, A., et al. 2010, MNRAS, 407, 83

Fabricant, D., Chilingarian, I., Hwang, H. S., et al. 2013, PASP, 125, 1362

Finkelstein, S. L. 2016, PASA, 33, e037

Flores-Cacho, I., Pierini, D., Soucail, G., et al. 2016, A\&A, 585, A54

Fowler, J. W., Niemack, M. D., Dicker, S. R., et al. 2007, ApOpt, 46, 3444

Fruchter, A. S., \& Hook, R. 2018, arXiv:astro-ph/9708242

Frye, B., \& Broadhurst, T. J. 1998, ApJ, 499, 115

Frye, B., Broadhurst, T., \& Benítez, N. 2002, ApJ, 568, 558

Frye, B. L., Bowen, D. V., Hurley, M., et al. 2008, ApJL, 685, L5

Frye, B. L., Coe, D., Bowen, D. V., et al. 2007, ApJ, 665, 921

Girardi, M., Biviano, A., Giuricin, G., Mardirossian, F., \& Mezzetti, M. 1993 , ApJ, 404, 38
Gladders, M. D., \& Yee, H. K. C. 2000, AJ, 120, 2148

Griffiths, A., Conselice, C. J., Alpaslan, M., et al. 2018, MNRAS, 475, 2853

Harrington, K. C., Yun, M. S., Cybulski, R., et al. 2016, MNRAS, 458, 4383

Harris, A. I., Baker, A. J., Frayer, D. T., et al. 2012, ApJ, 752, 152

Hasselfield, M., Hilton, M., Marriage, T. A., et al. 2013, JCAP, 7, 008

Hayashi, M., Kodama, T., Kohno, K., et al. 2017, ApJL, 841, L21

Hou, A., Parker, L. C., Harris, W. E., \& Wilman, D. J. 2009, ApJ, 702, 1199

Jaffé, Y. L., Smith, R., Candlish, G. N., et al. 2015, MNRAS, 448, 1715

Johnson, T. L., \& Sharon, K. 2016, ApJ, 832, 82

Kneissl, R., Polletta, M. D. C., Martinache, C., et al. 2018, arXiv:1804.06581

Koester, B. P., McKay, T. A., Annis, J., et al. 2007a, ApJ, 660, 221

Koester, B. P., McKay, T. A., Annis, J., et al. 2007b, ApJ, 660, 239

Lamarre, J. M., Puget, J. L., Bouchet, F., et al. 2003, NewAR, 47, 1017

Madau, P., \& Dickinson, M. 2014, ARA\&A, 52, 415

Mahler, G., Richard, J., Clément, B., et al. 2018, MNRAS, 473, 663

Mantz, A., Allen, S. W., Rapetti, D., \& Ebeling, H. 2010, MNRAS, 406, 1759

Martinache, C., Rettura, A., Dole, H., et al. 2018, A\&A, in press

Menanteau, F., Hughes, J. P., Sifón, C., et al. 2012, ApJ, 748, 7

Mihos, J. C. 2016, in IAU Symp. 317, The General Assembly of Galaxy Halos:

Structure, Origin and Evolution, ed. A. Bragaglia et al. (Cambridge: Cambridge Univ. Press), 27

Mo, H. J., \& White, S. D. M. 1996, MNRAS, 282, 347

Nayyeri, H., Keele, M., Cooray, A., et al. 2016, ApJ, 823, 17

Negrello, M., Amber, S., Amvrosiadis, A., et al. 2017, MNRAS, 465, 3558

Nesvadba, N., Kneissl, R., Cañameras, R., et al. 2016, A\&A, 593, L2

Peng, C. Y., Ho, L. C., Impey, C. D., \& Rix, H.-W. 2010, AJ, 139, 2097

Pilbratt, G. L., Riedinger, J. R., Passvogel, T., et al. 2010, A\&A, 518, L1

Planck Collaboration, 2015, A\&A, 582, A30

Planck Collaboration, 2016, A\&A, 596, A100

Planck Collaboration, Ade, P. A. R., Aghanim, N., et al. 2014, A\&A, 571, A20

Planck Collaboration, Ade, P. A. R., Aghanim, N., et al. 2016, A\&A, 594, A27

Planck Collaboration, Aghanim, N., Akrami, Y., et al. 2018, arXiv:1807.06209

Puget, J.-L., Abergel, A., Bernard, J.-P., et al. 1996, A\&A, 308, L5

Rabien, S., Angel, R., Barl, L., et al. 2018, A\&A, 621, A4

Rosati, P., Della Ceca, R., Norman, C., \& Giacconi, R. 1998, ApJL, 492, L21

Rozo, E., Wechsler, R. H., Rykoff, E. S., et al. 2010, ApJ, 708, 645

Rykoff, E. S., Rozo, E., Busha, M. T., et al. 2014, ApJ, 785, 104

Rykoff, E. S., Rozo, E., Hollowood, D., et al. 2016, ApJS, 224, 1

Sehgal, N., Addison, G., Battaglia, N., et al. 2013, ApJ, 767, 38

Sehgal, N., Trac, H., Acquaviva, V., et al. 2011, ApJ, 732, 44

Serra, P., Amblard, A., Temi, P., et al. 2011, ApJ, 740, 22

Siegel, S. R., Sayers, J., Mahdavi, A., et al. 2016, arXiv:1612.05377

Spilker, J. S., Bezanson, R., Marrone, D. P., et al. 2016, ApJ, 832, 19

The Astropy Collaboration, Price-Whelan, A. M., Sipőcz, B. M., et al. 2018, AJ, 156, 123

van Dokkum, P. G. 2001, PASP, 113, 1420

Vieira, J. D., Crawford, T. M., Switzer, E. R., et al. 2010, ApJ, 719, 763

Vieira, J. D., Marrone, D. P., Chapman, S. C., et al. 2013, Natur, 495, 344

Vikhlinin, A., Kravtsov, A. V., Burenin, R. A., et al. 2009, ApJ, 692, 1060

Wardlow, J. L., Cooray, A., De Bernardis, F., et al. 2013, ApJ, 762, 59

Weiß, A., De Breuck, C., Marrone, D. P., et al. 2013, ApJ, 767, 88

Windhorst, R. A., Cohen, S. H., Hathi, N. P., et al. 2011, ApJS, 193, 27

Windhorst, R. A., Timmes, F. X., Wyithe, J. S. B., et al. 2018, ApJS, 234, 41

Zitrin, A., Broadhurst, T., Umetsu, K., et al. 2009, MNRAS, 396, 1985

Zitrin, A., Labbé, I., Belli, S., et al. 2015, ApJL, 810, L12

Zitrin, A., Zheng, W., Broadhurst, T., et al. 2014, ApJL, 793, L12 